\title{
Physical Layer Network Coding for the $K$-user Multiple Access Relay Channel
}

\author{
Vijayvaradharaj T. Muralidharan and B. Sundar Rajan \\ Dept. of ECE, IISc, Bangalore 560012, India, Email:\{tmvijay, bsrajan\}@ece.iisc.ernet.in
}

\begin{abstract}
A Physical layer Network Coding (PNC) scheme is proposed for the $K$-user wireless Multiple Access Relay Channel (MARC), in which $K$ source nodes transmit their messages to the destination node $D$ with the help of a relay node $R$. The proposed PNC scheme involves two transmission phases: (i) Phase 1 during which the source nodes transmit, the relay node and the destination node receive and (ii) Phase 2 during which the source nodes and the relay node transmit, and the destination node receives. At the end of Phase 1 , the relay node decodes the messages of the source nodes and during Phase 2 transmits a many-to-one function of the decoded messages. Wireless networks in which the relay node decodes, suffer from loss of diversity order if the decoder at the destination is not chosen properly. A novel decoder is proposed for the PNC scheme, which offers the maximum possible diversity order of 2 , for a proper choice of certain parameters and the network coding map. Specifically, the network coding map used at the relay is chosen to be a $K$ dimensional Latin Hypercube, in order to ensure the maximum diversity order of 2 . Also, it is shown that the proposed decoder can be implemented by a fast decoding algorithm. Simulation results presented for the 3-user and 4-user MARC show that the proposed scheme offers a large gain over the existing scheme for the $K$-user MARC.
\end{abstract}

\section{BACKGROUND AND PRELIMINARIES}

We consider the $K$-user Multiple Access Relay Channel (MARC) shown in Fig. 1. Source nodes $S_{1}, S_{2}, \ldots, S_{K}$ want to transmit messages to the destination node $D$ with the help of the relay node $R$. All the nodes are assumed to have halfduplex constraint, i.e., the nodes cannot transmit and receive simultaneously in the same frequency band. In a $K$-user MARC, the maximum diversity order obtainable is two, since in addition to the presence of direct links, communication paths exist from the source nodes to the destination node $D$ through the relay node $R$.

\section{A. Background}

In a fading scenario, Multiple Input Multiple Output (MIMO) antenna systems provide gain in terms of spatial diversity. However, in many practical scenarios, it is difficult to place multiple collocated antennas in a single terminal. An attractive alternative to obtain diversity gain without using multiple antennas, is the utilization of intermediate relay nodes which aid the transmission from the source nodes to the destination nodes. In order to exploit the presence of intermediate relay nodes to obtain diversity gain, the source nodes need to convey their messages to the relay nodes. Due to the superposition nature of the wireless channel, if the source nodes transmit simultaneously in the same frequency band, interference occurs at the relay nodes. A loss of spectral efficiency results, if the source nodes transmit in orthogonal time/frequency slots. A solution to this problem is the use of physical layer network coding, first introduced in [1] , in which the nodes are allowed to transmit simultaneously. Physical layer Network Coding (PNC) has been shown to outperform traditional schemes which involve orthogonal transmissions [1]- [5]. So far, most of the works on PNC have mainly focussed only on the two-way relay channel. In our recent work [6], we proposed a scheme based on PNC for the two user MARC. In this paper, we present the generalization of the scheme proposed in [6] for the $K$-user MARC.

For the MARC, a Complex Field Network Coding (CFNC) scheme was proposed in [7]. The CFNC scheme, like the PNC scheme, avoids the loss of spectral efficiency, by making the source nodes transmit simultaneously. But the major difference between the CFNC scheme and the proposed PNC scheme is that during the relaying phase, the CFNC scheme uses a signal set of size $M^{K}$ at $R$, whereas the proposed PNC scheme uses a signal set of size $M$, where $M$ is the size of the signal set used at the source nodes.

As noted in [7], if the relay node transmits a many-toone function of the estimates of the messages transmitted by the source nodes and minimum squared Euclidean distance decoder is employed at $\mathrm{D}$, a loss of diversity order results. This problem of loss of diversity order due to error propagation, is encountered in many other wireless scenarios as well and various solutions have been proposed to avoid this problem. Cyclic Redundancy Check bits are used so that the nodes forward only those packets which are decoded correctly [8]. Some works assume the knowledge of all the instantaneous fade coefficients or error probabilities associated with the intermediate nodes at the destination node, with the decoder at the destination using this knowledge to ensure full diversity [9], [10]. The CFNC scheme proposed in [7], uses a scaling factor at the relay node which depends on the instantaneous fade coefficients associated with the links from the source nodes to the relay node, with the scaling factor indicated to the destination using pilot symbols. The proposed scheme does not suffer from the disadvantages of any of the above methods, yet ensures the maximum possible diversity order. This is achieved by means of an efficient choice of the transmission scheme and the use of a novel decoder at the destination $D$.

For the proposed PNC scheme, making the source nodes also transmit during the relaying phase, combined with a novel decoder ensures the maximum possible diversity order of two. Furthermore, if certain parameters are chosen properly, the 
proposed decoder for the PNC scheme can be implemented with a decoding complexity order same as that of the CFNC scheme proposed in [7].

For the two-way relay channel, the network coding maps used at the relay node need to form a mathematical structure called Latin Squares, for ensuring unique decodability at the end nodes [11]. The structural properties of Latin Squares have been used to obtain the network coding maps in a two-way relay channel [11]- [13]. Interestingly, choosing the network coding map used at $\mathrm{R}$ to be a $K$-dimensional Latin Hypercube, which is the generalization of the Latin Square to $K$ dimensions, helps towards achieving the maximum diversity order of two for the $K$-user MARC.

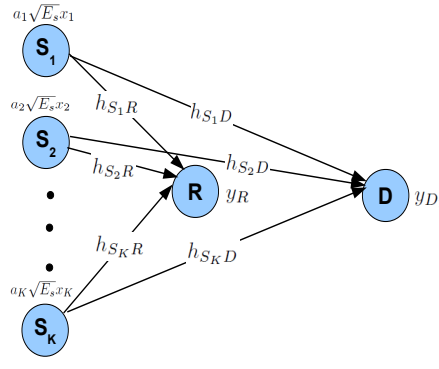

(a) Phase 1

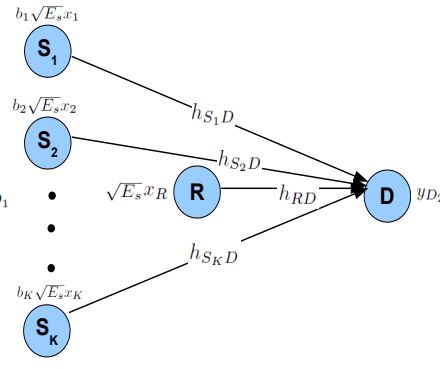

(b) Phase 2
Fig. 1: The two user Multiple Access Relay Channel

The main advantages of the proposed scheme over the CFNC scheme proposed in [7] are summarized below:

- In the CFNC scheme, $R$ transmits a complex linear combination of the estimate of the messages transmitted by the source node and the signal set used at $R$ during the relaying phase has $M^{K}$ points, where $M$ is the size of the signal set used at the source nodes. The minimum distance of the signal set used during the relaying phase vanishes as $K$ increases. In contrast, since the proposed PNC scheme uses a many-to-one map, the signal set used during the relaying phase has only $M$ points. The minimum distance of the signal set used at $R$ is more than that of the CFNC scheme and it remains the same irrespective of the number of source nodes $K$. Hence the proposed scheme performs better than the CFNC scheme. Simulation results presented for the 3-user and 4-user MARC confirm that the proposed PNC scheme provides a large gain over the CFNC scheme.

- In the CFNC scheme, $R$ uses a scaling factor which is a function of the instantaneous fade coefficients associated with the links from the source nodes to the relay node, which needs to be indicated to $D$ using pilot symbols. Since the proposed PNC scheme does not involve any such scaling factor, there is no need of such pilot symbols.

Notations: Throughout, vectors are denoted by bold lower case letters and matrices are denoted by bold capital letters. The set of complex numbers is denoted by $\mathbb{C}$. $\mathcal{C N}\left(0, \sigma^{2}\right)$ denotes a circularly symmetric complex Gaussian random variable with mean zero and variance $\sigma^{2}$ and $\mathcal{N}\left(0, \sigma^{2}\right)$ denotes a real Gaussian random variable with mean zero and variance $\sigma^{2}$. For a matrix $\mathbf{A}, \mathbf{A}^{\mathbf{T}}$ and $\mathbf{A}^{*}$ denotes its transpose and conjugate transpose respectively. For a complex number $x$, $x^{*}$ denotes its conjugate and $|x|$ denotes its absolute value. For a vector $\mathbf{v},\|\mathbf{v}\|$ denotes its Euclidean norm. The total transmission energy at a node is assumed to be equal to $E_{s}$ and all the additive noises are assumed to have a variance equal to 1 . By SNR, we denote the transmission energy $E_{s}$. For a signal set $\mathcal{S}, \Delta \mathcal{S}$ denotes the difference signal set of $\mathcal{S}, \Delta \mathcal{S}=\left\{x-x^{\prime} \mid x, x^{\prime} \in \mathcal{S}\right\}$. The all zero matrix of size $n \times n$ is denoted by $\mathbf{O}_{\mathbf{n}}$. The natural logarithm of $x$ is denoted by $\log (x) . \mathbb{E}(X)$ denotes the expectation of $X . Q[$.] denotes the complementary CDF of the standard Gaussian random variable.

\section{B. Signal Model}

Throughout, a quasi-static fading scenario is assumed with the channel state information available only at the receivers. The source nodes want to transmit a binary vector of length $\lambda$ to the destination node. At each one of the source nodes, the binary vector is mapped onto a point from a $M=2^{\lambda}$ point signal set denoted by $\mathcal{S}$. Let $\mu: \mathbb{F}_{2}^{\lambda} \rightarrow \mathcal{S}$ denote the mapping from bits to complex symbols used at the source nodes.

The proposed PNC scheme involves two transmission phases: Phase 1 during which the source nodes simultaneously transmit and, $R$ and $D$ receive, followed by the Phase 2 during which the source nodes and $R$ transmit to $D$.

Phase 1: Let $x_{i}=\mu\left(s_{i}\right) \in \mathcal{S}, i \in\{1,2 \ldots, K\}, s_{i} \in \mathbb{F}_{2}^{\lambda}$ denote the complex symbol the source node $S_{i}$ wants to convey to $D$. During Phase 1, the source node $S_{i}$ transmits a scaled version of $x_{i}$. The received signal at $R$ and $D$ during Phase 1 are respectively given by,

$$
\begin{aligned}
& y_{R}=\sum_{i=1}^{K} h_{S_{i} R} \sqrt{E_{s}} a_{i} x_{i}+z_{R} \text { and } \\
& y_{D_{1}}=\sum_{i=1}^{K} h_{S_{i} D} \sqrt{E_{s}} a_{i} x_{i}++z_{D_{1}},
\end{aligned}
$$

where $a_{i} \in \mathbb{C}, i \in\{1,2 \ldots, K\}$ are constants and the additive noises $z_{R}$ and $z_{D_{2}}$ are assumed to be $\mathcal{C N}(0,1)$. All the fade coefficients are Rayleigh distributed, with the fade coefficient associated with the $S_{i}-R$ link $h_{S_{i} R} \sim \mathcal{C N}\left(0, \sigma_{S_{i} R}^{2}\right)$, and the fade coefficient associated with the $S_{i}-D$ link $h_{S_{i} D} \sim$ $\mathcal{C N}\left(0, \sigma_{S_{i} D}^{2}\right)$.

Based on the received complex number $y_{R}$, the relay node computes the Maximum Likelihood (ML) estimate of $\left(x_{1}, x_{2}, \ldots, x_{K}\right)$ denoted by $\left(\hat{x}_{1}^{R}, \hat{x}_{2}^{R}, \ldots, \hat{x}_{K}^{R}\right)$, i.e.,

$\left(\hat{x}_{1}^{R}, \hat{x}_{2}^{R}, \ldots, \hat{x}_{K}^{R}\right)=\arg \min _{\left(x_{1}^{\prime}, x_{2}^{\prime}, \ldots, x_{K}^{\prime}\right) \in \mathcal{S}^{K}}\left|y_{R}-\sum_{i=1}^{K} h_{S_{i} R} \sqrt{E_{s}} a_{i} x_{i}^{\prime}\right|$.

Phase 2: During Phase 2, the source node $S_{i}$ transmits a scaled version of $x_{i}$ and $R$ transmits $x_{R}=$ $f\left(\hat{x}_{1}^{R}, \hat{x}_{2}^{R}, \ldots, \hat{x}_{K}^{R}\right)$, where $f: \mathcal{S}^{K} \rightarrow \mathcal{S}$ is a many-to-one function. The received signal at $D$ during Phase 2 is given by, 


$$
y_{D_{2}}=\sum_{i=1}^{K} h_{S_{i} D} \sqrt{E_{s}} b_{i} x_{i}+h_{R D} \sqrt{E_{s}} x_{R}+z_{D_{2}},
$$

where $b_{i} \in \mathbb{C}$ are constants and the additive noise $z_{D_{2}}$ is assumed to be $\mathcal{C N}(0,1)$. The fade coefficient associated with the $R-D$ link $h_{R D}$ is assumed to be $\mathcal{C N}\left(0, \sigma_{R D}^{2}\right)$.

For the transmission energy at the source nodes to be equal to $E_{s}$, the constants $a_{i}$ and $b_{i}$ are chosen such that $\left|a_{i}\right|^{2}+$ $\left|b_{i}\right|^{2}=1, \forall i \in\{1,2 \ldots, K\}$.

From (1) and (2), the received complex numbers at $D$ during the two phases can be written in vector form as,

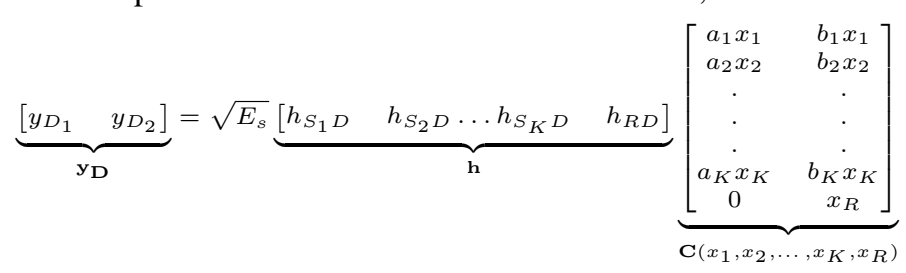

$$
\begin{aligned}
& +\underbrace{\left[\begin{array}{ll}
z_{D_{1}} & z_{D_{2}}
\end{array}\right]}_{\mathbf{z}_{\mathbf{D}}}
\end{aligned}
$$

The matrix $\mathbf{C}\left(x_{1}, x_{2}, \ldots, x_{K}, x_{R}\right)$ in (3) is referred to as the codeword matrix. The restriction of $\mathbf{C}\left(x_{1}, x_{2}, \ldots, x_{K}, x_{R}\right)$ to the first $K$ rows, denoted by $\mathbf{C}_{\mathbf{r}}\left(x_{1}, x_{2}, \ldots, x_{K}\right)$ is referred to as the restricted codeword matrix, i.e., $\mathbf{C}_{\mathbf{r}}\left(x_{1}, x_{2}, \ldots, x_{K}\right)=\left[\begin{array}{llll}a_{1} x_{1} & a_{2} x_{2} & \ldots & a_{K} x_{K} \\ b_{1} x_{1} & b_{2} x_{2} & \ldots & b_{K} x_{K}\end{array}\right]^{T}$. The difference between any two restricted codeword matrices is referred as the restricted codeword difference matrix, i.e., the restricted codeword difference matrices are of the form $\mathbf{C}_{\mathbf{r}}\left(\Delta x_{1}, \Delta x_{2}, \ldots, \Delta x_{K}\right)=\left[\begin{array}{llll}a_{1} \Delta x_{1} & a_{2} \Delta x_{2} & \ldots & a_{K} \Delta x_{K} \\ b_{1} \Delta x_{1} & b_{2} \Delta x_{2} & \ldots & b_{K} \Delta x_{K}\end{array}\right]^{T}$, where $\Delta x_{i} \in \Delta \mathcal{S}, \forall i \in\{1,2, \ldots, K\}$.

From (3), the vector $\mathbf{y}_{\mathbf{D}}$ can also be written as,

$$
\mathbf{y}_{\mathbf{D}}=\sum_{i=1}^{K} \sqrt{E_{s}} x_{i} \mathbf{h} \mathbf{W}_{\mathbf{i}}+\sqrt{E_{s}} x_{R} \mathbf{h} \mathbf{W}_{\mathbf{R}}+\mathbf{z}_{\mathbf{D}}
$$

where $\mathbf{W}_{\mathbf{i}}$ is a $(K+1) \times 2$ matrix whose $i^{\text {th }}$ row is given by $\left[a_{i} b_{i}\right]$ and all other entries are zeros. For the $(K+1) \times 2$ matrix $\mathbf{W}_{\mathbf{R}}$, the $(K+1)^{\text {th }}$ row is given by $\left[\begin{array}{ll}0 & 1\end{array}\right]$ and all other entries are zeros. The matrices $\mathbf{W}_{\mathbf{i}}, i \in\{1,2 \ldots, K\}$ and $\mathbf{W}_{\mathbf{R}}$ are referred to as the weight matrices.

The contributions and organization of the paper are as follows: A novel decoder for the proposed PNC scheme is presented in Section II A. In Section II B, it is shown that the decoder presented in Section II A achieves the maximum diversity order of two if the following two conditions are satisfied: (i) the map $f$ used at the relay node forms a $K$ dimensional Latin Hypercube and (ii) the constants $a_{i}$ and $b_{i}, i \in\{1,2 \ldots, K\}$ are such that every $2 \times 2$ square submatrix of the restricted codeword difference matrices have rank two when $\Delta x_{i}$ takes non-zero values. In Section III, the condition under which the proposed decoder admits fast decoding is obtained. It is shown that when at least one of the weight matrices $\mathbf{W}_{\mathbf{i}}$ is Hurwitz-Radon orthogonal with $\mathbf{W}_{\mathbf{R}}$, the proposed decoder admits fast decoding, with the decoding complexity order same as that of the CFNC scheme proposed in [7]. Simulation results which show that the proposed PNC scheme provides large gain over the CFNC scheme are presented in Section IV.

\section{A Novel Decoder for the Proposed PNC Scheme AND ITS DIVERSITY ANALYSIS}

In the following subsection, a novel decoder for the proposed PNC scheme is presented.

\section{A. A Novel Decoder for the Proposed PNC Scheme}

When $D$ uses the minimum squared Euclidean distance decoder given by,

$$
\begin{aligned}
& \left(\hat{x}_{1}^{D}, \hat{x}_{2}^{D}, \ldots, \hat{x}_{K}^{D}\right)=\arg \underset{\left(x_{1}, x_{2}, \ldots, x_{K}\right) \in \mathcal{S}^{K}}{\min }\left\{\left|y_{D_{1}}-\sum_{i=1}^{K} h_{S_{i} D} \sqrt{E_{s}} a_{i} x_{i}\right|^{2}\right. \\
& \left.+\left|y_{D_{2}}-\sum_{i=1}^{K} h_{S_{i} D} \sqrt{E_{s}} b_{i} x_{i}-h_{R D} \sqrt{E_{s}} f\left(x_{1}, x_{2}, \ldots, x_{K}\right)\right|^{2}\right\},
\end{aligned}
$$

a loss of diversity order results, since this decoder does not consider the possibility of decoding errors at the relay node.

Alternatively, we propose a novel decoder given by,

$$
\begin{array}{r}
\left(\hat{x}_{1}^{D}, \hat{x}_{2}^{D}, \ldots, \hat{x}_{K}^{D}\right)=\underset{\left(x_{1}, x_{2}, \ldots, x_{K}\right) \in \mathcal{S}^{K}}{\arg }\left\{m_{1}\left(x_{1}, x_{2}, \ldots, x_{K}\right),\right. \\
\left.\log (S N R)+m_{2}\left(x_{1}, x_{2}, \ldots, x_{K}\right)\right\},
\end{array}
$$

where the metrics $m_{1}$ and $m_{2}$ are given in (5) and (6) respectively, at the top of the next page.

The idea behind the choice of this decoder is as follows: The optimal ML decoding metric at $D$ is equal to $m_{1}\left(x_{1}, x_{2}, \ldots, x_{K}\right)$, when the relay transmits the correct network-coded symbol. The relay transmits a wrong networkcoded symbol, independent of $\left(x_{1}, x_{2}, \ldots, x_{K}\right)$, if the joint ML estimate at the relay $\left(\hat{x}_{1}^{R}, \hat{x}_{2}^{R}, \ldots, \hat{x}_{K}^{R}\right)$ is such that $x_{R}=f\left(\hat{x}_{1}^{R}, \hat{x}_{2}^{R}, \ldots, \hat{x}_{K}^{R}\right) \neq f\left(x_{1}, x_{2}, \ldots, x_{K}\right)$. Under this condition, the optimal ML decision metric at $D$ is given by $m_{2}\left(x_{1}, x_{2}, \ldots, x_{K}\right)$. At high $\mathrm{SNR}$, the relay transmits a wrong network-coded symbol with a probability which is proportional to $\frac{1}{S N R}$. Hence, to the metric $m_{2}\left(x_{1}, x_{2}, \ldots, x_{K}\right)$, we add a correction factor of $\log (S N R)$ and the minimum of $m_{1}\left(x_{1}, x_{2}, \ldots, x_{K}\right)$ and $\log (S N R)+m_{2}\left(x_{1}, x_{2}, \ldots, x_{K}\right)$ is taken to be the decoding metric at $D$.

The CFNC scheme proposed in [7] uses the minimum squared Euclidean distance decoder, which has a decoding complexity of $\mathcal{O}\left(M^{K}\right)$. Since the decoder given in (4) involves minimization over $K+1$ variables $x_{1}, x_{2}, \ldots x_{K}$ and $x_{R}$, it appears as though the decoding complexity order is $\mathcal{O}\left(M^{K+1}\right)$. In Section III, it is shown that by properly choosing the constants $a_{i}$ 's and $b_{i}$ 's, the decoding complexity order can be reduced to $\mathcal{O}\left(M^{K}\right)$ which is the same as that of the CFNC scheme.

\section{B. Diversity Analysis of the Proposed Decoder}

The following theorem gives a sufficient condition under which the decoder given in (4) offers maximum diversity order two.

Theorem 1: For the proposed PNC scheme, the decoder given in (4) offers maximum diversity order two if the following two conditions are satisfied:

1) The map $f$ satisfies the condition, 


$$
\begin{aligned}
& m_{1}\left(x_{1}, x_{2}, \ldots, x_{K}\right)=\left|y_{D_{1}}-\sum_{i=1}^{K} h_{S_{i} D} \sqrt{E_{s}} a_{i} x_{i}\right|^{2}+\left|y_{D_{2}}-\sum_{i=1}^{K} h_{S_{i} D} \sqrt{E_{s}} b_{i} x_{i}-h_{R D} \sqrt{E_{s}} f\left(x_{1}, x_{2}, \ldots, x_{K}\right)\right|^{2} \\
& m_{2}\left(x_{1}, x_{2}, \ldots, x_{K}\right)=\left|y_{D_{1}}-\sum_{i=1}^{K} h_{S_{i} D} \sqrt{E_{s}} a_{i} x_{i}\right|^{2}+{ }_{x_{R} \neq f\left(x_{1}, x_{2}, \ldots, x_{K}\right), x_{R} \in \mathcal{S}}\left\{\left.\left|y_{D_{2}}-\sum_{i=1}^{K} h_{S_{i} D} \sqrt{E_{s}} b_{i} x_{i}-h_{R D} \sqrt{E_{s}} x_{R}\right|\right|^{2}\right\} \\
& m_{3}\left(x_{1}, x_{2}, \ldots, x_{K}\right)=\left|y_{D_{1}}-\sum_{i=1}^{K} h_{S_{i} D} \sqrt{E_{s}} a_{i} x_{i}\right|^{2}+\min _{x_{R} \in \mathcal{S}}\left\{\left|y_{D_{2}}-\sum_{i=1}^{K} h_{S_{i} D} \sqrt{E_{s}} b_{i} x_{i}-h_{R D} \sqrt{E_{s}} x_{R}\right|^{2}\right\}
\end{aligned}
$$

$$
\begin{aligned}
f\left(x_{1}, x_{2}, \ldots,\right. & \left.x_{i-1}, x_{i}, x_{i+1}, \ldots, x_{K}\right) \\
& \neq f\left(x_{1}, x_{2}, \ldots, x_{i-1}, x_{i}^{\prime}, x_{i+1}, \ldots, x_{K}\right),
\end{aligned}
$$

for $x_{i} \neq x_{i}^{\prime}$, for all $i \in\{1,2, \ldots, K\}$.

2) All $2 \times 2$ submatrices of the restricted codeword difference matrices $\mathbf{C}_{\mathbf{r}}\left(\Delta x_{1}, \Delta x_{2}, \ldots, \Delta x_{K}\right)$ have rank two, $\forall \Delta x_{1}, \Delta x_{2}, \ldots, \Delta x_{K} \neq 0$.

It is easy to verify that a map $f$ satisfying condition 1) above forms a Latin Hypercube of order $M$ and dimension $K$.

Definition 1: [14] A Latin Hypercube of order $M$ and dimension $K$ is an array of dimension $K$, with the indices for the $K$ dimensions as well as the entries filled in the array taking values from the symbol set $\{0,1 \ldots, M-1\}$. Every symbol occurs exactly once along each one of the $K$ dimensions.

The $i^{\text {th }}$ dimension of the Latin Hypercube represents the transmission $x_{i}$ of the source node $S_{i}$. For simplicity, the points of the $M$ point signal set $\mathcal{S}$ are indexed by integers from 0 to $M-1$. The entries filled in the Latin Hypercube represent the transmission of the relay node.

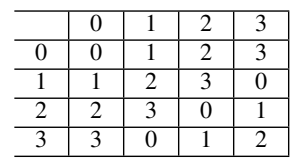

Fig. 2: An example of Latin Square of order 4

A Latin Hypercube of dimension 2 is a Latin Square. Fig. 2 shows an example of a Latin Square of order 4. It can be seen from Fig. 2 that no two entries repeat in a row as well as a column.
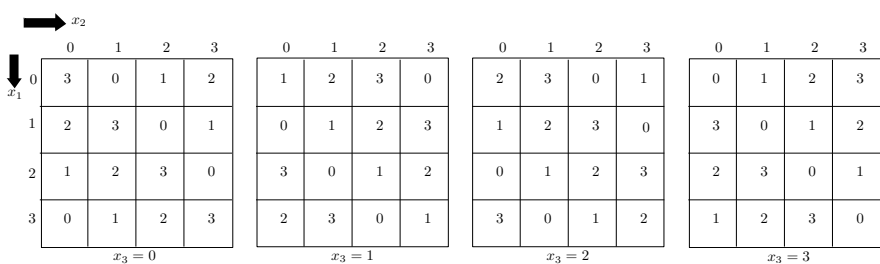

Fig. 3: An example of Latin Cube of order 4

A Latin Hypercube of dimension 3 is a Latin Cube. The three dimensions of a Latin Cube are referred to as rows, columns and pages. Fig. 3 shows an example of a Latin Cube of order 4. It can be verified from Fig. 3 that in each one of the four pages, no two entries repeat in a row as well as a column.
Similarly, for a fixed value of row index and a fixed value of column index, the entries in the four pages are distinct.

$$
\begin{aligned}
& \rightarrow x_{2} x_{3}=0 \quad x_{3}=1 \quad x_{3}=2 \quad x_{3}=3
\end{aligned}
$$

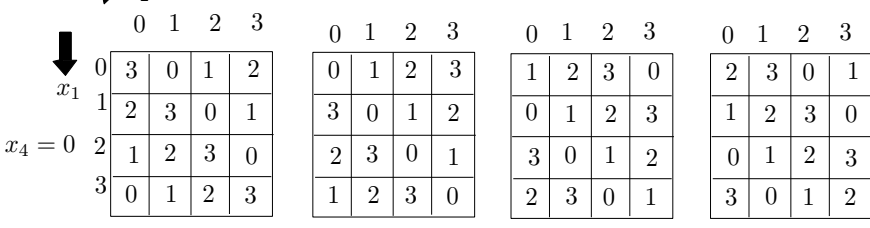

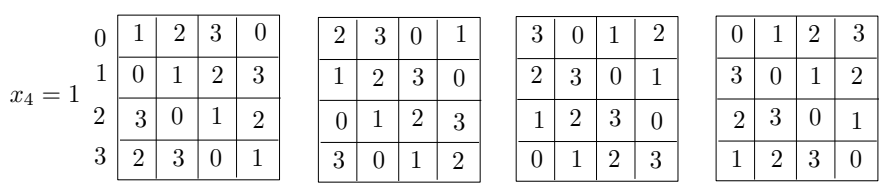

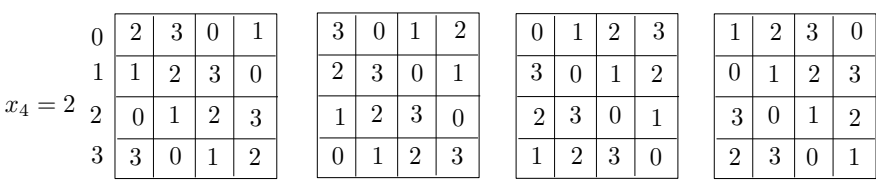

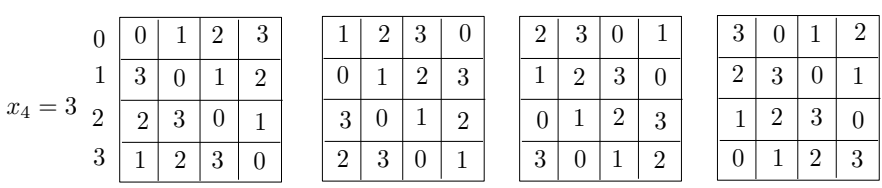

Fig. 4: An example of Latin Hypercube of dimension 4 and order 4

Fig. 4 shows a Latin Hypercube of dimension 4 and order 4. In Fig. 4 with a $4 \times 4$ square for the which the indices of the third and fourth dimensions are fixed, no two entries repeat in a row as well as a column. Also, when the first three dimensions are fixed, no two entries repeat when the fourth dimension is varied. Similarly, fixing the first, second and fourth dimensions, the four entries obtained when the third dimension is varied are distinct.

For the 3-user MARC, the following example gives a choice of $a_{i}$ 's and $b_{i}$ 's for which Condition 2) given in Theorem 1 is satisfied.

Example 1: For the 3-user MARC, choosing $a_{1}=1$, $b_{1}=0, a_{2}=\frac{1}{\sqrt{2}}, b_{2}=\frac{1}{\sqrt{2}}, a_{3}=\frac{1}{\sqrt{2}}$ and $b_{3}=-\frac{1}{\sqrt{2}}$, the restricted codeword difference matrices are of the form $\mathbf{C}_{\mathbf{r}}\left(\Delta x_{1}, \Delta x_{2}, \Delta x_{3}\right)=\left[\begin{array}{cc}\Delta x_{1} & 0 \\ \frac{1}{\sqrt{2}} \Delta x_{2} & \frac{1}{\sqrt{2}} \Delta x_{2} \\ \frac{1}{\sqrt{2}} \Delta x_{3} & -\frac{1}{\sqrt{2}} \Delta x_{3}\end{array}\right]$. It can be verified that when $\Delta x_{1}, \Delta x_{2}$ and $\Delta x_{3}$ take non-zero values, the rank of every $2 \times 2$ square submatrix of $\mathbf{C}_{\mathbf{r}}\left(\Delta x_{1}, \Delta x_{2}, \Delta x_{3}\right)$ is two and hence condition 2) given in Theorem 1 is satisfied. 
Example 2: For the 4-user MARC, choosing $a_{1}=1, b_{1}=$ $0, a_{2}=\frac{1}{\sqrt{2}}, b_{2}=\frac{1}{\sqrt{2}}, a_{3}=\frac{1}{\sqrt{2}}, b_{3}=-\frac{1}{\sqrt{2}}, a_{4}=\frac{j}{\sqrt{2}}$ and $b_{4}=\frac{1}{\sqrt{2}}$, the restricted codeword difference matrices are of the form $\mathbf{C}_{\mathbf{r}}\left(\Delta x_{1}, \Delta x_{2}, \Delta x_{3}, \Delta x_{4}\right)=\left[\begin{array}{cc}\Delta x_{1} & 0 \\ \frac{1}{\sqrt{2}} \Delta x_{2} & \frac{1}{\sqrt{2}} \Delta x_{2} \\ \frac{1}{\sqrt{2}} \Delta x_{3} & -\frac{1}{\sqrt{2}} \Delta x_{3} \\ \frac{j}{\sqrt{2}} \Delta x_{4} & \frac{1}{\sqrt{2}} \Delta x_{4}\end{array}\right]$. It can be verified that when $\Delta x_{1}, \Delta x_{2}, \Delta x_{3}$ and $\Delta x_{4}$ take nonzero values, the rank of every $2 \times 2$ square submatrix of $\mathbf{C}_{\mathbf{r}}\left(\Delta x_{1}, \Delta x_{2}, \Delta x_{3}, \Delta x_{4}\right)$ is two and hence condition 2) given in Theorem 1 is satisfied.

In general, for the $K$-user MARC, there are many possible ways of choosing $a_{i}$ 's and $b_{i}$ 's so that condition 2) given in Theorem 1 is satisfied. Choosing $K$ unit-norm vectors $\left[\begin{array}{ll}a_{i} & b_{i}\end{array}\right], i \in\{1,2, \ldots, K\}$, from $\mathbb{C}^{2}$ such that $\left[a_{i} b_{i}\right] \neq$ $c\left[a_{j} b_{j}\right], c \in \mathbb{C}$, for all $i \neq j$ ensures that condition 2) given in Theorem 1 is satisfied. One particular choice of $a_{i}$ 's and $b_{i}$ 's which satisfies the above condition for the $K$-user MARC is given in the next example.

Example 3: Consider the set of vectors over $\mathbb{C}^{2}$ given by $V=\left\{\left[\cos (\theta) e^{j \phi} \sin (\theta)\right],\left[-\sin (\theta) \cos (\theta) e^{-j \phi}\right]: 0<\theta<\frac{\pi}{2},-\pi \leq \phi<\pi\right\}$.

For the $K$-user MARC, choosing $\left[\begin{array}{ll}a_{1} & b_{1}\end{array}\right]=\left[\begin{array}{ll}0 & 0\end{array}\right]$ and $\left[a_{i} b_{i}\right], i \in\{2,3, \ldots, K\}$, to be any $K-1$ distinct vectors from the set $V$ ensures that condition 2) given in Theorem 1 is satisfied.

\section{A Fast Decoding Algorithm for the Proposed DECODER}

In this section, it is shown that if the constants $a_{i}$ 's and $b_{i}$ 's are chosen properly, the decoder given in (4) can be implemented using an efficient algorithm with a complexity order $\mathcal{O}\left(M^{K}\right)$. Note that for the CFNC scheme proposed in [7], the decoding complexity order at $D$ is $\mathcal{O}\left(M^{K}\right)$.

Before the algorithm is presented, some notations are introduced. The points in the signal set $\mathcal{S}$ are denoted by $s_{i}, 1 \leq i \leq M$.

From (3), the vector $\mathbf{y}_{\mathbf{D}}^{\mathbf{T}}$ can be written as,

$$
\mathbf{y}_{\mathbf{D}}^{\mathbf{T}}=\underbrace{\left[\begin{array}{lllll}
a_{1} h_{S_{1} D} & a_{2} h_{S_{2} D} & \ldots & a_{K} h_{S_{K} D} & 0 \\
b_{1} h_{S_{1} D} & b_{2} h_{S_{2} D} & \ldots & b_{K} h_{S_{K} D} & h_{R D}
\end{array}\right]}_{\mathbf{H}_{\mathbf{e q}}} \underbrace{\left[\begin{array}{c}
x_{1} \\
x_{2} \\
\vdots \\
\vdots \\
x_{K} \\
x_{R}
\end{array}\right]}_{\mathbf{x}} \sqrt{E_{s}}+\mathbf{z}_{\mathbf{D}}^{\mathbf{T}} .
$$

The matrix $\mathbf{H}_{\mathbf{e q}}$ can be decomposed using $\mathbf{Q R}$ decomposition as $\mathbf{H}_{\mathbf{e q}}=\mathbf{Q R}$, where $\mathbf{Q}$ is a $2 \times 2$ unitary matrix and $\mathbf{R}=$ $\left[\begin{array}{ll}\mathbf{R}_{\mathbf{1}} & \mathbf{R}_{\mathbf{2}}\end{array}\right]$ is a $2 \times(K+1)$ matrix, with $\mathbf{R}_{\mathbf{1}}$ being uppertriangular of size $2 \times 2$ and $\mathbf{R}_{\mathbf{2}}$ being a $2 \times(K-1)$ matrix. Let $r_{i j}$ denote the $(i, j)^{t h}$ entry of $\mathbf{R}$.

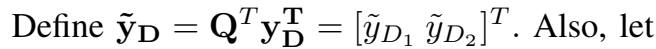

$$
\begin{aligned}
\phi_{1}\left(x_{1}, x_{2}, \ldots, x_{K}\right)= & \left|\tilde{y}_{D_{1}}-\sum_{i=1}^{K} r_{1 i} x_{i} \sqrt{E_{s}}\right|^{2}, \\
\phi_{2}\left(x_{1}, x_{2}, \ldots, x_{K}\right)=\mid \tilde{y}_{D_{2}}-\sum_{i=2}^{K} r_{2 i} x_{i} \sqrt{E_{s}} & \quad-\left.r_{2(K+1)} f\left(x_{1}, x_{2}, \ldots, x_{K}\right) \sqrt{E_{s}}\right|^{2}, \\
\phi_{3}\left(x_{2}, \ldots, x_{K}, x_{R}\right)= & \left|\tilde{y}_{D_{2}}-\sum_{i=2}^{K} r_{2 i} x_{i} \sqrt{E_{s}}-r_{2(K+1)} x_{R} \sqrt{E_{s}}\right|^{2} .
\end{aligned}
$$

The following proposition gives a sufficient condition under which Algorithm 1 below implements the decoder given in (4).

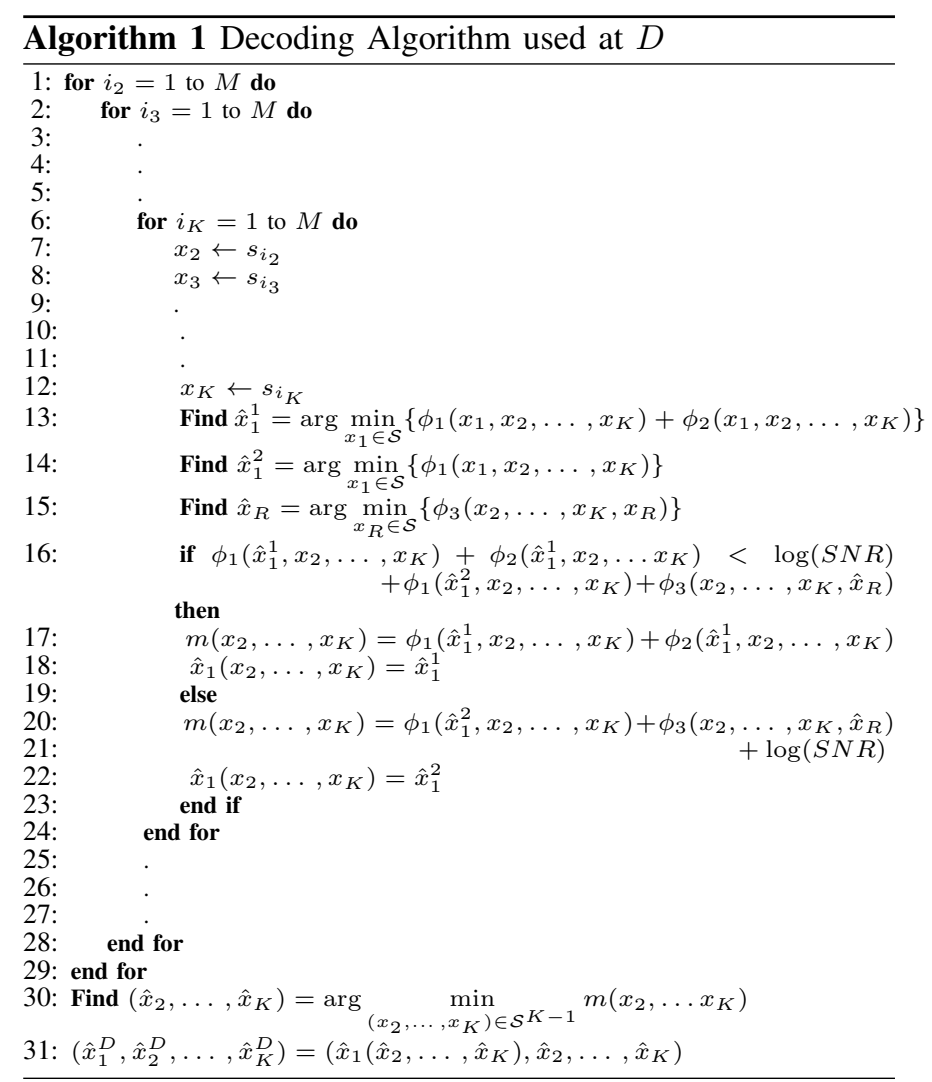

Proposition 1: Algorithm 1 1 implements the decoder in (4), if the constants $a_{i}$ 's and $b_{i}$ 's are such that the weight matrices $\mathbf{W}_{\mathbf{1}}$ and $\mathbf{W}_{\mathbf{R}}$ are Hurwitz-Radon (H-R) orthogonal, i.e., $\mathbf{W}_{\mathbf{1}} \mathbf{W}_{\mathbf{R}}{ }^{*}+\mathbf{W}_{\mathbf{R}} \mathbf{W}_{\mathbf{1}}{ }^{*}=\mathbf{O}_{\mathbf{K}+\mathbf{1}}$.

Proof: The decoding metric of the decoder given in (4) can be written as,

$$
\begin{aligned}
& \min _{\left(x_{1}, x_{2}, \ldots, x_{K}\right)}\left\{m_{1}\left(x_{1}, x_{2}, \ldots, x_{K}\right), \log (S N R)+m_{2}\left(x_{1}, x_{2}, \ldots, x_{K}\right)\right\} \\
& =\min _{\left(x_{1}, x_{2}, \ldots, x_{K}\right)}\left\{m_{1}\left(x_{1}, x_{2}, \ldots, x_{K}\right),\right. \\
& \left.\log (S N R)+m_{1}\left(x_{1}, x_{2}, \ldots, x_{K}\right), \log (S N R)+m_{2}\left(x_{1}, x_{2}, \ldots, x_{K}\right)\right\} . \\
& =\min _{\left(x_{1}, x_{2}, \ldots, x_{K}\right)}\left\{m_{1}\left(x_{1}, x_{2}, \ldots, x_{K}\right),\right. \\
& \left.\log (S N R)+m_{3}\left(x_{1}, x_{2}, \ldots, x_{K}\right)\right\} .
\end{aligned}
$$

${ }^{1}$ A algorithm exactly similar to Algorithm 1 can be used with the roles of $S_{1}$ and $S_{i}$ interchanged, if $\mathbf{W}_{\mathbf{i}}$ and $\mathbf{W}_{\mathbf{R}}$ are H-R orthogonal. 
where the metric $m_{3}\left(x_{1}, x_{2}, \ldots, x_{K}\right)$ is given in (7), at the top of the previous to the previous page. We have,

$$
\begin{aligned}
m_{3}\left(x_{1}, x_{2}, \ldots, x_{K}\right) & =\min _{x_{R} \in \mathcal{S}}\left\{\left\|\mathbf{y}_{\mathbf{D}}^{T}-\mathbf{H}_{\mathbf{e q}} \mathbf{x} \sqrt{E_{s}}\right\|^{2}\right\} \\
& =\min _{x_{R} \in \mathcal{S}}\left\{\left\|\tilde{\mathbf{y}}_{\mathbf{D}}-\mathbf{R} \mathbf{x} \sqrt{E_{s}}\right\|^{2}\right\} .
\end{aligned}
$$

Since $\mathbf{R}_{\mathbf{1}}$ is upper triangular, $r_{21}=0$. Also, the entry $r_{1(K+1)}=0$, since $\mathbf{W}_{\mathbf{A}}$ and $\mathbf{W}_{\mathbf{R}}$ are $\mathrm{H}-\mathbf{R}$ orthogonal (follows from Theorem 2, [15]). Hence, from (10], it follows that,

$$
\begin{gathered}
m_{3}\left(x_{1}, x_{2}, \ldots, x_{K}\right)=\left|\tilde{y}_{D_{1}}-\sum_{i=1}^{K} r_{1 i} x_{i} \sqrt{E_{s}}\right|^{2} \\
+\min _{x_{R} \in \mathcal{S}}\left\{\left|\tilde{y}_{D_{2}}-\sum_{i=2}^{K} r_{2 i} x_{i} \sqrt{E_{s}}-r_{2(K+1)} x_{R} \sqrt{E_{s}}\right|^{2}\right\} .
\end{gathered}
$$

Hence,

$$
\begin{aligned}
\min _{x_{1} \in \mathcal{S}} m_{3}\left(x_{1}, x_{2}, \ldots, x_{K}\right) & =\min _{x_{1} \in \mathcal{S}} \phi_{1}\left(x_{1}, x_{2}, \ldots, x_{K}\right) \\
& +\min _{x_{R} \in \mathcal{S}} \phi_{3}\left(x_{2}, \ldots, x_{K}, x_{R}\right) .
\end{aligned}
$$

From (9), the decoding metric can be written as,

$$
\begin{aligned}
& \min _{\left(x_{2}, \ldots, x_{K}\right) \in \mathcal{S}^{K-1}}\left\{\operatorname { m i n } _ { x _ { 1 } \in \mathcal { S } } \left\{\phi_{1}\left(x_{1}, x_{2}, \ldots, x_{K}\right)\right.\right. \\
&\left.+\phi_{2}\left(x_{1}, x_{2}, \ldots, x_{K}\right)\right\}, \\
& \min _{x_{1} \in \mathcal{S}} \phi_{1}\left(x_{1}, x_{2}, \ldots, x_{K}\right) \\
&\left.\quad+\min _{x_{R} \in \mathcal{S}} \phi_{3}\left(x_{2}, \ldots, x_{K}, x_{R}\right)+\log (S N R)\right\} .
\end{aligned}
$$

In Algorithm 1, inside the $K-1$ nested for loops, the values of $x_{2}, x_{3} \ldots, x_{K}$ are fixed and the operations in lines 13,14 and 15 involve a complexity order $\mathcal{O}(M)$. The operations from line 16 to line 23 involve constant complexity, independent of $M$. Hence the complexity order for executing the nested for loops from line 1 to line 29 is $\mathcal{O}\left(M^{K}\right)$. The operation in line 30 involves a complexity order $\mathcal{O}\left(M^{K-1}\right)$. Hence the overall complexity order of Algorithm 1 is $\mathcal{O}\left(M^{K}\right)$, which is the same as that of the CFNC scheme proposed in [7].

Example 4: Continuing with Example 1, for the 3-user MARC, when $a_{1}=1, b_{1}=0, a_{2}=\frac{1}{\sqrt{2}}, b_{2}=\frac{1}{\sqrt{2}}$ and $a_{3}=\frac{1}{\sqrt{2}}$, and $b_{3}=-\frac{1}{\sqrt{2}}$, the weight matrices are given by, $\mathbf{W}_{\mathbf{1}}=\left[\begin{array}{llll}1 & 0 & 0 & 0 \\ 0 & 0 & 0 & 0\end{array}\right]^{T}, \mathbf{W}_{\mathbf{2}}=\left[\begin{array}{cccc}0 & \frac{1}{\sqrt{2}} & 0 & 0 \\ 0 & \frac{1}{\sqrt{2}} & 0 & 0\end{array}\right]^{T}$, $\mathbf{W}_{\mathbf{3}}=\left[\begin{array}{cccc}0 & 0 & \frac{1}{\sqrt{2}} & 0 \\ 0 & 0 & -\frac{1}{\sqrt{2}} & 0\end{array}\right]^{T}$ and $\mathbf{W}_{\mathbf{R}}=\left[\begin{array}{cccc}0 & 0 & 0 & 0 \\ 0 & 0 & 0 & 1\end{array}\right]^{T}$. It can be verified that the matrices $\mathbf{W}_{\mathbf{1}}$ and $\mathbf{W}_{\mathbf{R}}$ are H-R orthogonal, i.e., $\mathbf{W}_{\mathbf{1}} \mathbf{W}_{\mathbf{R}}{ }^{*}+\mathbf{W}_{\mathbf{R}} \mathbf{W}_{\mathbf{1}}{ }^{*}=\mathbf{O}_{\mathbf{4}}$. Hence, for this case, Algorithm 1 can be used to implement the decoder given in (4).

\section{Simulation Results}

Simulation results presented in this section compare the performance of the proposed PNC scheme with the CFNC scheme proposed in [7], for the 3-user and 4-user MARC. In the simulation results presented for 3-user MARC, the values of the constants $a_{i}$ 's and $b_{i}$ 's are chosen to be the ones in Example 1 with 4-PSK signal set is used at the nodes and the Latin Cube given in Fig. 3 is used as the network coding map at the relay node. For the 4-user MARC, the values of the constants $a_{i}$ 's and $b_{i}$ 's are chosen to be the ones in Example 2 with 4-PSK signal set is used at the nodes and the Latin Hypercube of dimension 4 given in Fig. 4 is used as the network coding map at the relay node.

For the 3-user MARC, for the case when the variances of all the fading links are $0 \mathrm{~dB}$, the SNR Vs. Symbol Error Probability (SEP) plots are shown in Fig. 5. It can be seen from Fig. 5 that the PNC scheme performs better than the CFNC scheme and offers a large gain of $8 \mathrm{~dB}$, when the SEP is $10^{-4}$. Fig. 6 shows a similar plot for the case when $\sigma_{S_{i} R}^{2}=10$ $\mathrm{dB}$ and $\sigma_{S_{i} D}^{2}=\sigma_{R D}^{2}=0 \mathrm{~dB}$, where $i \in\{1,2,3\}$. It can be seen from Fig. 6 that for this case, the PNC scheme offers a gain of nearly $6 \mathrm{~dB}$, when the SEP is $10^{-4}$. Fig. 7 shows the plots for the case when the $R-D$ link is stronger than all other links, i.e, $\sigma_{S_{i} R}^{2}=\sigma_{S_{i} D}^{2}=\sigma_{B D}^{2}=0 \mathrm{~dB}, i \in\{1,2,3\}$ and $\sigma_{R D}^{2}=10 \mathrm{~dB}$. For this case, the PNC scheme offers a large gain of about $12 \mathrm{~dB}$, when the SEP is $10^{-4}$. Also, it can be verified from the plots that the proposed decoder for the PNC scheme offers the maximum possible diversity order of two.

Fig. 8 . Fig. 9 and Fig. 10 show similar plots for the 4-user MARC with 4-PSK signal set. When the variances of all the fading links are $0 \mathrm{~dB}$, from Fig. 8 it can be seen that the proposed PNC scheme offers a gain of $13 \mathrm{~dB}$, when the SEP is $10^{-4}$. For the case when $\sigma_{S_{i} R}^{2}=10, i \in\{1,2,3\}$, and all other variances are $0 \mathrm{~dB}$, from Fig. 9 it can be seen that the PNC scheme offers a gain of nearly $7.5 \mathrm{~dB}$, when the SEP is $10^{-4}$. For the case when the link from $R$ to $D$ is stronger the other links by $10 \mathrm{~dB}$, it can be seen from Fig. 10 that the PNC scheme offers an advantage of $17 \mathrm{~dB}$ over the CFNC scheme.

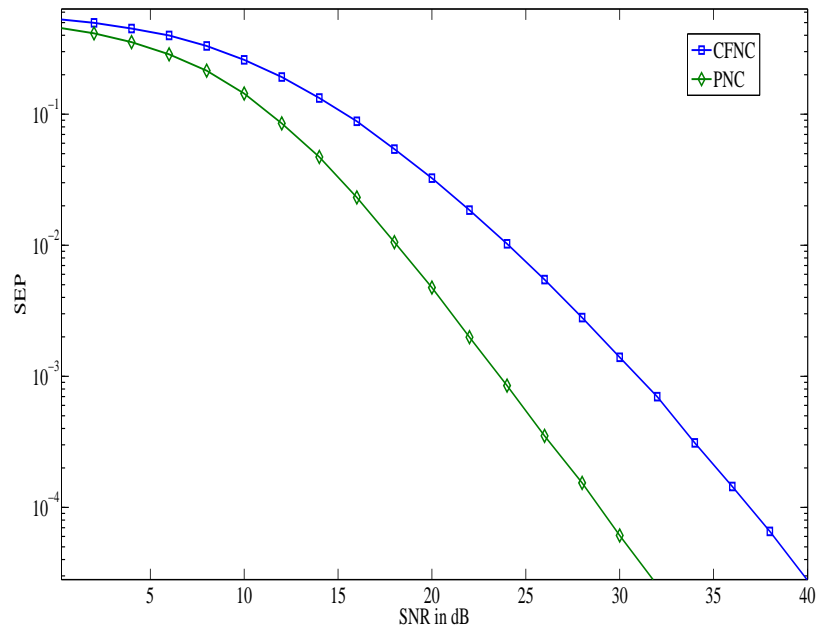

Fig. 5: SNR vs SEP plots for 4-PSK signal set for $\sigma_{S_{i} R}^{2}=$ $\sigma_{S_{i} D}^{2}=\sigma_{R D}^{2}=0 \mathrm{~dB}$ for the 3-user MARC with 4-PSK signal set. 


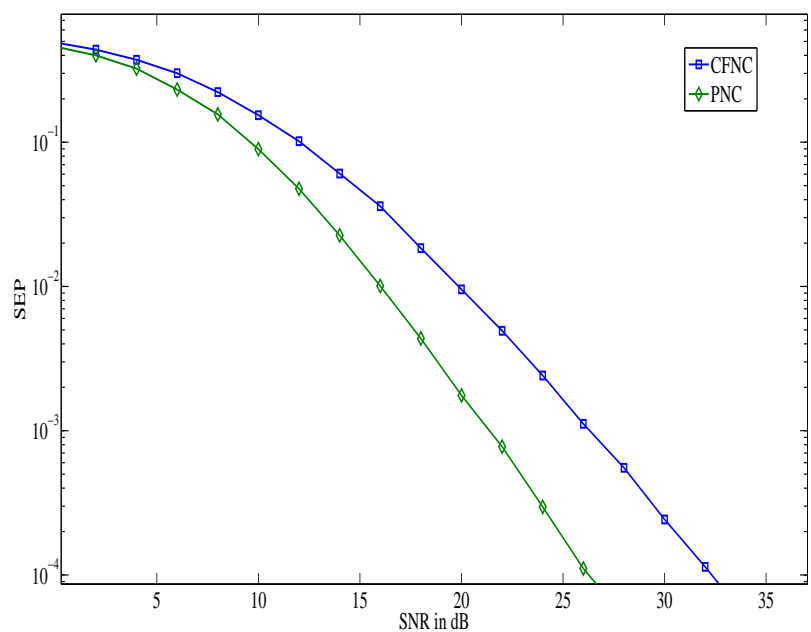

Fig. 6: SNR vs SEP plots for $\sigma_{S_{i} R}^{2}=10 \mathrm{~dB}, \sigma_{S_{i} D}^{2}=\sigma_{B D}^{2}=$ $\sigma_{R D}^{2}=0 \mathrm{~dB}$ for the 3-user MARC with 4-PSK signal set.

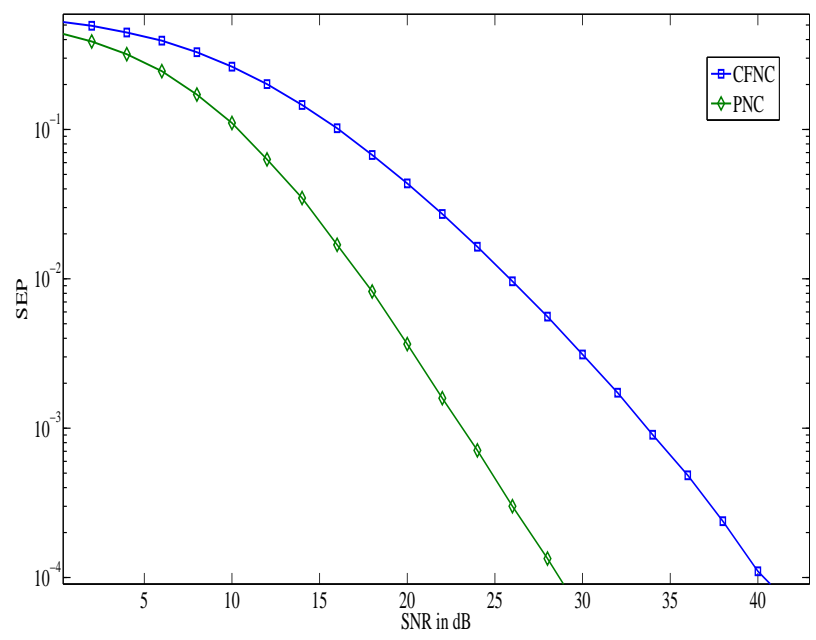

Fig. 7: SNR vs SEP plots for 4-PSK signal set for $\sigma_{S_{i} R}^{2}=$ $\sigma_{S_{i} D}^{2}=0 \mathrm{~dB}, \sigma_{R D}^{2}=10 \mathrm{~dB}$ for the 3-user MARC with 4-PSK signal set.

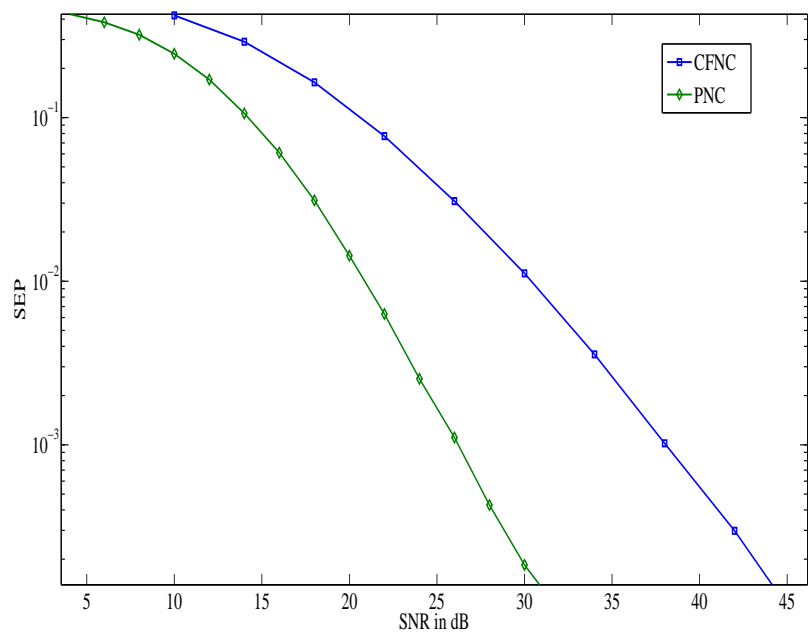

Fig. 8: SNR vs SEP plots for 4-PSK signal set for $\sigma_{S_{i} R}^{2}=$ $\sigma_{S_{i} D}^{2}=\sigma_{R D}^{2}=0 \mathrm{~dB}$ for the 4-user MARC with 4-PSK signal set

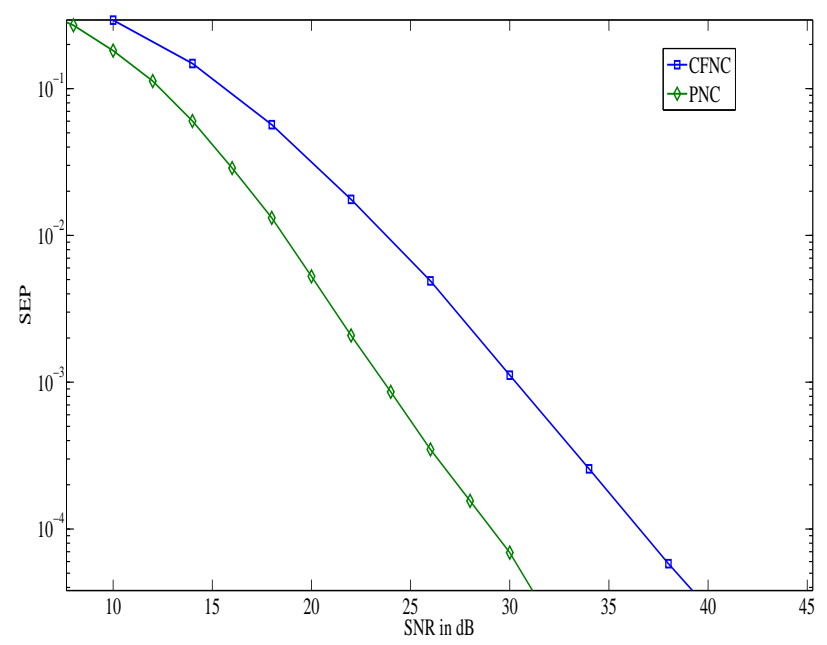

Fig. 9: SNR vs SEP plots for 4-PSK signal set for $\sigma_{S_{i} R}^{2}=10$ $\mathrm{dB}, \sigma_{S_{i} D}^{2}=\sigma_{B D}^{2}=\sigma_{R D}^{2}=0 \mathrm{~dB}$ for the 4-user MARC with 4-PSK signal set.

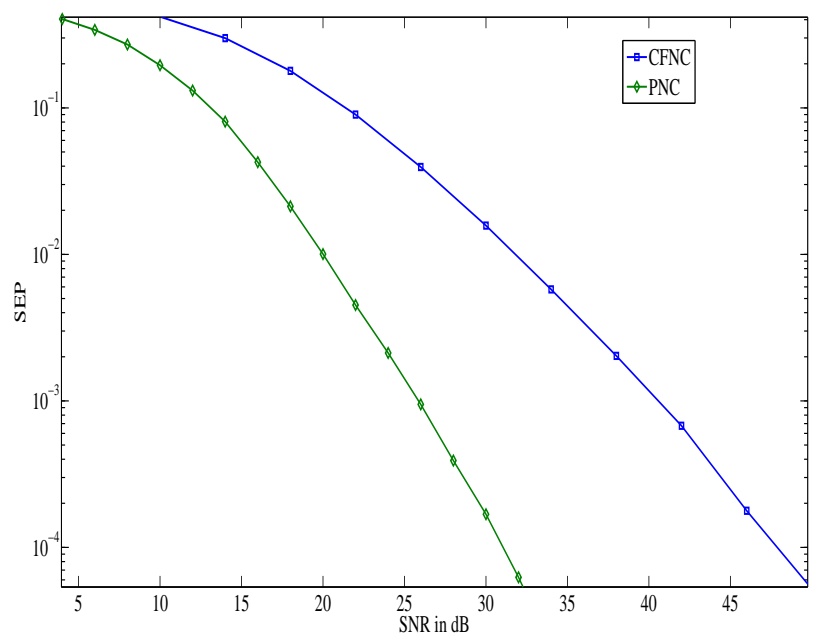

Fig. 10: SNR vs SEP plots for 4-PSK signal set for $\sigma_{S_{i} R}^{2}=$ $\sigma_{S_{i} D}^{2}=0 \mathrm{~dB}, \sigma_{R D}^{2}=10 \mathrm{~dB}$ for the 4-user MARC with 4-PSK signal set

\section{DisCusSION}

A physical layer network coding scheme was proposed for the $K$-user Multiple Access Relay Channel. For the proposed scheme, a novel decoder was presented and it was shown that the decoder offers the maximum possible diversity order of two if the network coding map used at the relay forms a $K$-dimensional Latin Hypercube and every $2 \times 2$ submatrix of a restricted codeword difference matrix $\mathbf{C}_{\mathbf{r}}\left(\Delta x_{1}, x_{2}, \ldots, \Delta x_{K}\right), \Delta x_{i} \neq 0, \forall i \in\{1,2, \ldots, K\}$, has a rank two. Also, it was shown that the proposed decoder can be implemented using a fast decoding algorithm, if a weight matrix $\mathbf{W}_{\mathbf{i}}$ is Hurwitz-Radon orthogonal with $\mathbf{W}_{\mathbf{R}}$, for some $i \in\{1,2 \ldots, K\}$. The problem of finding the constants $a_{i}$ 's and $b_{i}$ 's which minimize the error probability in addition to ensuring maximum diversity order remains open. Extension of the proposed scheme for the case when there are multiple 
relay nodes is a possible direction for future work.

\section{REFERENCES}

[1] S. Zhang, S. C. Liew and P. P. Lam, "Hot topic: Physical-layer network coding," in Proc. ACM Annual Int. Conf. Mobile Computing and Networking, Los Angeles, 2006, pp. 358-365.

[2] P. Popovski and H. Yomo, "The anti-packets can increase the achievable throughput of a wireless multihop network," in Proc. IEEE Int. Conf. Communications, Istanbul, 2006, pp. 3885-3890.

[3] P. Popovski and H. Yomo, "Physical network coding in two-Way wireless relay channels," in Proc. IEEE Int. Conf. Communications, Glasgow, 2007, pp. 707-712.

[4] M. P. Wilson, K. R. Narayanan, H. D. Pfister, and A. Sprintson, "Joint physical layer coding and network coding for bi-directional relaying," IEEE Trans. Info. Theory, vol. 56, pp. 5641-5654, Nov. 2010.

[5] T. Koike-Akino, P. Popovski and V. Tarokh, "Optimized constellation for two-way wireless relaying with physical network coding," IEEE J. Sel. Areas Commun., vol. 27, pp. 773-787, June 2009.

[6] Vijayvaradharaj T. Muralidharan and B. Sundar Rajan, "Physical Layer Network Coding for the Multiple Access Relay Channel," available online at arXiv: 1210.0490 [cs.IT], Oct. 2012.

[7] T. Wang and G. B. Giannakis, "Complex field network coding for multiuser cooperative communications," IEEE J. Sel. Areas Commun., vol. 26, pp. 561-571, April 2008

[8] M. Janani, A. Hedayat, T. Hunter, and A. Nosratinia, "Coded cooperation in wireless communications: space-time transmission and iterative decoding," IEEE Trans. Signal Process., vol. 52, pp. 362-371, Feb. 2004.

[9] T. Wang, A. Cano, G. B. Giannakis and J. N. Laneman, "Highperformance cooperative demodulation with Decode-and-Forward relays," IEEE Trans. Commun., vol. 5, pp.1427-1438, July 2007.

[10] M. Ju and I.- M. Kim, "ML performance analysis of the Decodeand-Forward protocol in cooperative diversity networks," IEEE Trans. Wireless Commun., vol. 8, pp. 3855-3867, July 2009.

[11] V. Namboodiri, V. T. Muralidharan and B. S. Rajan, "Wireless bidirectional relaying and Latin Squares," in Proc. IEEE Wireless Communications and Networking Conf., Paris, 2012, pp. 1404-1409 (a detailed version is available in arXiv: $1110.0084 \mathrm{v} 2$ [cs.IT], 16 Nov. 2011).

[12] V. T. Muralidharan and B. S. Rajan, "Wireless network coding for MIMO two-way relaying using Latin Rectangles," in Proc. IEEE Int. Symp. Inf. Theory, Cambridge, 2012.

[13] V. Namboodiri and B. S. Rajan, "Wirless network coding for QAM bidirectional relaying and Latin Squares," in Proc. IEEE Global Telecommunications Conference, Anaheim, 2012.

[14] K. Kishen, "On Latin and Hyper-Graeco-Latin Cubes and Hyper Cubes," Current Science, vol. 11, pp. 98-99, 1942.

[15] K. P. Srinath and B. S. Rajan, "Low ML Decoding Complexity, Large Coding Gain, Full Rate, Full-Diversity STBCs for $2 \times 2$ and $4 \times$ 2 MIMO systems," IEEE J. Sel. Topics Signal Process., vol. 3, pp. 916-927, December 2009.

[16] V. Tarokh, N. Seshadri and A. R. Calderbank, "Space-time codes for high data rate wireless communication: Performance criterion and code construction," IEEE Trans. Info. Theory, vol. 44, pp. 744-765, March 1998.

\section{APPENDIX - PROOF OF THEOREM 1}

Let $H$ denote a particular realization of the fade coefficients. Throughout the proof, the subscript $H$ in a probability expression indicates conditioning on the fade coefficients. For simplicity of notation, it is assumed that the variances of all the fading coefficients are one, but the result holds for other values as well.

Let $E$ denote an error event that the transmitted message $K$-tuple $\left(x_{1}, x_{2}, \ldots, x_{K}\right)$ is wrongly decoded at D.

The probability of $E$ conditioned on $H$ given in (12), can be upper bounded as in 113) (eqns. (12) and (13) are shown at the next page). $P_{H}\left\{f\left(\hat{x}_{1}^{R}, \hat{x}_{2}^{R}, \ldots, \hat{x}_{K}^{R}\right)=f\left(x_{1}, x_{2}, \ldots, x_{K}\right)\right\} \quad$ and $P_{H}\left\{f\left(\hat{x}_{1}^{R}, \hat{x}_{2}^{R}, \ldots, \hat{x}_{K}^{R}\right) \neq f\left(x_{1}, x_{2}, \ldots, x_{K}\right)\right\}$ respectively denote the probabilities that $R$ transmits the correct and wrong network coded symbol during Phase 2 , for a given $H$. Also, the probability $P_{H}\left\{\left(\hat{x}_{1}^{D}, \hat{x}_{2}^{D}, \ldots, \hat{x}_{K}^{D}\right) \neq\left(x_{1}, x_{2}, \ldots, x_{K}\right)\right.$ $\left.\mid f\left(\hat{x}_{1}^{R}, \hat{x}_{2}^{R}, \ldots, \hat{x}_{K}^{R}\right)=f\left(x_{1}, x_{2}, \ldots, x_{K}\right)\right\} \quad$ and the probability $P_{H}\left\{\left(\hat{x}_{1}^{D}, \hat{x}_{2}^{D}, \ldots, \hat{x}_{K}^{D}\right) \neq\left(x_{1}, x_{2}, \ldots, x_{K}\right)\right.$ $\left.\mid f\left(\hat{x}_{1}^{R}, \hat{x}_{2}^{R}, \ldots, \hat{x}_{K}^{R}\right) \neq f\left(x_{1}, x_{2}, \ldots, x_{K}\right)\right\} \quad$ in respectively denote the probabilities of $E$ given that $R$ transmitted the correct and wrong network coded symbol for a given $H . P_{H}\{E\}$ can be upper bounded as in (14), where $P_{H}\left\{f\left(\hat{x}_{1}^{R}, \hat{x}_{2}^{R}, \ldots, \hat{x}_{K}^{R}\right)=x_{R}^{\prime}\right\}$ denotes the probability that the network coded symbol transmitted by $R$ is $x_{R}^{\prime} \neq f\left(x_{1}, x_{2}, \ldots, x_{K}\right)$ and the probability $P_{H}\left\{\left(\hat{x}_{1}^{D}, \hat{x}_{2}^{D}, \ldots, \hat{x}_{K}^{D}\right) \neq\left(x_{1}, x_{2}, \ldots, x_{K}\right)\right.$ $\left.\mid f\left(\hat{x}_{1}^{R}, \hat{x}_{1}^{R}, \ldots, \hat{x}_{K}^{R}\right)=x_{R}^{\prime}\right\}$ is the probability of $E$ given that $\mathrm{R}$ transmits $x_{R}^{\prime}$, for a given $H$. Taking expectation of the terms in (14) w.r.t $H$, we get (15).

The rest of the proof of Theorem 1 is presented in two parts as Lemma 1 and Lemma 2. In Lemma 1, it is shown that the probability $\quad P\left\{\left(\hat{x}_{1}^{D}, \hat{x}_{2}^{D}, \ldots, \hat{x}_{K}^{D}\right) \neq\left(x_{1}, x_{2}, \ldots, x_{K}\right)\right.$ $\left.\mid f\left(\hat{x}_{1}^{R}, \hat{x}_{2}^{R}, \ldots, \hat{x}_{K}^{R}\right)=f\left(x_{1}, x_{2}, \ldots, x_{K}\right)\right\} \quad$ has a diversity order two. Lemma 2 shows that the probability $P\left\{\left(\hat{x}_{1}^{D}, \hat{x}_{2}^{D}, \hat{x}_{K}^{D}\right) \neq\left(x_{1}, x_{2}, \ldots, x_{K}\right)\right.$ $\left.\mid f\left(\hat{x}_{1}^{R}, \hat{x}_{2}^{R}, \ldots, \hat{x}_{K}^{R}\right)=x_{R}^{\prime}\right\}$ has a diversity order one. Since $P\left\{f\left(\hat{x}_{1}^{R}, \hat{x}_{2}^{R}, \ldots, \hat{x}_{K}^{R}\right)=x_{R}^{\prime}\right\}$ has a diversity order one, Lemma 1 and Lemma 2 together imply that $P\{E\}$ has a diversity order two.

Lemma 1: When the two conditions in the statement of Therorem 1 are satisfied, the probability $\quad P\left\{\left(\hat{x}_{1}^{D}, \hat{x}_{2}^{D}, \ldots \hat{x}_{K}^{D}\right) \neq\left(x_{1}, x_{2}, \ldots, x_{K}\right)\right.$ $\left.\mid f\left(\hat{x}_{1}^{R}, \hat{x}_{2}^{R}, \hat{x}_{K}^{R}\right)=f\left(x_{1}, x_{2}, \ldots, x_{K}\right)\right\}$ has a diversity order two.

Proof:

Recall that the decoder used at D given in (4) in Section II A, involves computation of the metrics $m_{1}$ and $m_{2}$ defined in (5) and (6). Under the condition that $R$ transmitted the correct network coding symbol, a decoding error occurs at $D$ only when $m_{1}\left(x_{1}, x_{2}, \ldots, x_{K}\right)>m_{1}\left(x_{1}^{\prime}, x_{2}^{\prime}, \ldots, x_{K}^{\prime}\right)$ or $m_{1}\left(x_{1}, x_{2}, \ldots, x_{K}\right)>\log (S N R)+m_{2}\left(x_{1}^{\prime}, x_{2}^{\prime}, \ldots, x_{K}^{\prime}\right)$ for some $\left(x_{1}^{\prime}, x_{2}, \ldots, x_{K}^{\prime}\right) \neq\left(x_{1}, x_{2}, \ldots, x_{K}\right)$. Hence, the probability $P\left\{\left(\hat{x}_{1}^{D}, \hat{x}_{2}^{D}, \ldots, \hat{x}_{K}^{D}\right) \neq\left(x_{1}, x_{2}, \ldots, x_{K}\right)\right.$ $\left.f\left(\hat{x}_{1}^{R}, \hat{x}_{2}^{R}, \ldots, \hat{x}_{K}^{R}\right)=f\left(x_{1}, x_{2}, \ldots, x_{K}\right)\right\} \quad$ can be upper bounded as in (16), which can be upper bounded using the union bound as in (17) (eqns. (16) and (17) are given at the next page).

The probability $P\left\{m_{1}\left(x_{1}, x_{2}, \ldots, x_{K}\right)>m_{1}\left(x_{1}^{\prime}, x_{2}^{\prime}, \ldots, x_{K}^{\prime}\right)\right.$ $\left.\mid f\left(\hat{x}_{1}^{R}, \hat{x}_{2}^{R}, \ldots, \hat{x}_{K}^{R}\right)=f\left(x_{1}, x_{2}, \ldots, x_{K}\right)\right\}$ is equal to the Pairwise Error Probability (PEP) of a space time coded $3 \times 1$ collocated MISO system, with the codeword difference matrices of the space time code used at the transmitter being of the form $\left[\begin{array}{lllll}a_{1} \Delta x_{1} & a_{2} \Delta x_{2} & \ldots & a_{K} \Delta x_{K} & 0 \\ b_{1} \Delta x_{1} & b_{2} \Delta x_{2} & \ldots & b_{K} \Delta x_{K} & \Delta x_{R}\end{array}\right]^{T}$, where $\Delta x_{i}=x_{i}-x_{i}^{\prime}, i \in\{1,2, \ldots, K$ and $\Delta x_{R}=f\left(x_{1}, x_{2}, \ldots, x_{K}\right)-f\left(x_{1}^{\prime}, x_{2}^{\prime}, \ldots, x_{K}^{\prime}\right)$. When $\Delta x_{i} \neq 0$, for at least two values of $i \in\{1,2, \ldots, K\}$, these codeword difference matrices are of rank 2, other wise condition 1) given in the statement of 


$$
\begin{aligned}
& P_{H}\{E\}=P_{H}\left\{\left(\hat{x}_{1}^{D}, \hat{x}_{2}^{D}, \ldots, \hat{x}_{K}^{D}\right) \neq\left(x_{1}, x_{2}, \ldots, x_{K}\right) \mid f\left(\hat{x}_{1}^{R}, \hat{x}_{2}^{R}, \ldots, \hat{x}_{K}^{R}\right)=f\left(x_{1}, x_{2}, \ldots, x_{K}\right)\right\} P_{H}\left\{f\left(\hat{x}_{1}^{R}, \hat{x}_{2}^{R}, \ldots, \hat{x}_{K}^{R}\right)=f\left(x_{1}, x_{2}, \ldots, x_{K}\right)\right\} \\
& +P_{H}\left\{\left(\hat{x}_{1}^{D}, \hat{x}_{2}^{D}, \ldots, \hat{x}_{K}^{D}\right) \neq\left(x_{1}, x_{2}, \ldots, x_{K}\right) \mid f\left(\hat{x}_{1}^{R}, \hat{x}_{2}^{R}, \ldots, \hat{x}_{K}^{R}\right) \neq f\left(x_{1}, x_{2}, \ldots, x_{K}\right)\right\} P_{H}\left\{f\left(\hat{x}_{1}^{R}, \hat{x}_{2}^{R}, \ldots, \hat{x}_{K}^{R}\right) \neq f\left(x_{1}, x_{2}, \ldots, x_{K}\right)\right\} \\
& \leq P_{H}\left\{\left(\hat{x}_{1}^{D}, \hat{x}_{2}^{D}, \ldots, \hat{x}_{K}^{D}\right) \neq\left(x_{1}, x_{2}, \ldots, x_{K}\right) \mid f\left(\hat{x}_{1}^{R}, \hat{x}_{2}^{R}, \ldots, \hat{x}_{K}^{R}\right)=f\left(x_{1}, x_{2}, \ldots, x_{K}\right)\right\} \\
& +P_{H}\left\{\left(\hat{x}_{1}^{D}, \hat{x}_{2}^{D}, \ldots, \hat{x}_{K}^{D}\right) \neq\left(x_{1}, x_{2}, \ldots, x_{K}\right) \mid f\left(\hat{x}_{1}^{R}, \hat{x}_{2}^{R}, \ldots, \hat{x}_{K}^{R}\right) \neq f\left(x_{1}, x_{2}, \ldots, x_{K}\right)\right\} P_{H}\left\{f\left(\hat{x}_{1}^{R}, \hat{x}_{2}^{R}, \ldots, \hat{x}_{K}^{R}\right) \neq f\left(x_{1}, x_{2}, \ldots, x_{K}\right)\right\} \\
& \leq P_{H}\left\{\left(\hat{x}_{1}^{D}, \hat{x}_{2}^{D}, \ldots, \hat{x}_{K}^{D}\right) \neq\left(x_{1}, x_{2}, \ldots, x_{K}\right) \mid f\left(\hat{x}_{1}^{R}, \hat{x}_{2}^{R}, \ldots, \hat{x}_{K}^{R}\right)=f\left(x_{1}, x_{2}, \ldots, x_{K}\right)\right\} \\
& +\sum_{\substack{f\left(\hat{x}_{1}^{R}, \hat{x}_{2}^{R}, \ldots, \hat{x}_{K}^{R}\right)=x_{R}^{\prime}, x_{R}^{\prime} \neq f\left(x_{1}, x_{2}, \ldots, x_{K}\right)}} P_{H}\left\{\left(\hat{x}_{1}^{D}, \hat{x}_{2}^{D}, \ldots, \hat{x}_{K}^{D}\right) \neq\left(x_{1}, x_{2}, \ldots, x_{K}\right) \mid f\left(\hat{x}_{1}^{R}, \hat{x}_{2}^{R}, \ldots, \hat{x}_{K}^{R}\right)=x_{R}^{\prime}\right\} P_{H}\left\{f\left(\hat{x}_{1}^{R}, \hat{x}_{2}^{R}, \ldots, \hat{x}_{K}^{R}\right)=x_{R}^{\prime}\right\} \\
& P\{E\} \leq P\left\{\left(\hat{x}_{1}^{D}, \hat{x}_{2}^{D}, \ldots, \hat{x}_{K}^{D}\right) \neq\left(x_{1}, x_{2}, \ldots, x_{K}\right) \mid f\left(\hat{x}_{1}^{R}, \hat{x}_{2}^{R}, \ldots, \hat{x}_{K}^{R}\right)=f\left(x_{1}, x_{2}, \ldots, x_{K}\right)\right\} \\
& \underset{\substack{f\left(\hat{x}_{1}^{R}, \hat{x}_{2}^{R}, \ldots, \hat{x}_{K}^{R}\right)=x_{R}^{\prime}, x_{R}^{\prime} \neq f\left(x_{1}, x_{2}, \ldots, x_{K}\right)}}{P}\left\{\left(\hat{x}_{1}^{D}, \hat{x}_{2}^{D}, \ldots, \hat{x}_{K}^{D}\right) \neq\left(x_{1}, x_{2}, \ldots, x_{K}\right) \mid f\left(\hat{x}_{1}^{R}, \hat{x}_{2}^{R}, \ldots, \hat{x}_{K}^{R}\right)=x_{R}^{\prime}\right\} P\left\{f\left(\hat{x}_{1}^{R}, \hat{x}_{2}^{R}, \ldots, \hat{x}_{K}^{R}\right)=x_{R}^{\prime}\right\} .
\end{aligned}
$$

$$
\begin{aligned}
& P\left\{\left(\hat{x}_{1}^{D}, \hat{x}_{2}^{D}, \ldots, \hat{x}_{K}^{D}\right) \neq\left(x_{1}, x_{2}, \ldots, x_{K}\right) \mid f\left(\hat{x}_{1}^{R}, \hat{x}_{2}^{R}, \ldots, \hat{x}_{K}^{R}\right)=f\left(x_{1}, x_{2}, \ldots, x_{K}\right)\right\} \\
& =P\left\{\left\{m_{1}\left(x_{1}, x_{2}, \ldots, x_{K}\right)>m_{1}\left(x_{1}^{\prime}, x_{2}^{\prime}, \ldots, x_{K}^{\prime}\right),\left(x_{1}^{\prime}, x_{2}^{\prime}, \ldots, x_{K}^{\prime}\right) \neq\left(x_{1}, x_{2}, \ldots, x_{K}\right)\right\}\right. \\
& \left.\cup\left\{m_{1}\left(x_{1}, x_{2}, \ldots, x_{K}\right)>\log (S N R)+m_{2}\left(x_{1}^{\prime}, x_{2}^{\prime}, \ldots, x_{K}^{\prime}\right),\left(x_{1}^{\prime}, x_{2}^{\prime}, \ldots, x_{K}^{\prime}\right) \neq\left(x_{1}, x_{2}, \ldots, x_{K}\right)\right\} \mid f\left(\hat{x}_{1}^{R}, \hat{x}_{2}^{R}, \ldots, \hat{x}_{K}^{R}\right)=f\left(x_{1}, x_{2}, \ldots, x_{K}\right)\right\} \\
& \leq \sum_{\substack{\left(x_{1}^{\prime}, x_{2}^{\prime}, \ldots, x_{K}^{\prime}\right) \in \mathcal{S}^{K} \\
\left(x_{1}, x_{2}^{\prime}, \ldots, x_{K}^{\prime}\right) \neq\left(x_{1}, x_{2}, \ldots, x_{K}\right)}}^{P}\left\{m_{1}\left(x_{1}, x_{2}, \ldots, x_{K}\right)>m_{1}\left(x_{1}^{\prime}, x_{2}^{\prime}, \ldots, x_{K}^{\prime}\right) \mid f\left(\hat{x}_{1}^{R}, \hat{x}_{2}^{R}, \hat{x}_{K}^{R}\right)=f\left(x_{1}, x_{2}, \ldots, x_{K}\right)\right\} \\
& \quad+\sum_{\left(x_{1}^{\prime}, x_{2}^{\prime}, \ldots, x_{K}^{\prime}\right) \neq\left(x_{1}, x_{2}, \ldots, x_{K}\right)} P\left\{m_{1}\left(x_{1}, x_{2}, \ldots, x_{K}\right)>\log (S N R)+m_{2}\left(x_{1}^{\prime}, x_{2}^{\prime}, \ldots, x_{K}^{\prime}\right) \mid f\left(\hat{x}_{1}^{R}, \hat{x}_{2}^{R}, \ldots, \hat{x}_{K}^{R}\right)=f\left(x_{1}, x_{2}, \ldots, x_{K}\right)\right\} \cdot(17) \\
&
\end{aligned}
$$

Theorem 1) will be violated. When $\Delta x_{i} \neq 0$ and $\Delta x_{j}=0, \forall i \neq j, i, j \in\{1,2, \ldots, K\}$, the codeword difference matrices are full rank, otherwise condition 2) in the statement of Theorem 1 will be violated. Since the codeword difference matrices are full rank, the probability $\quad P\left\{m_{1}\left(x_{1}, x_{2}, \ldots, x_{K}\right)>m_{1}\left(x_{1}^{\prime}, x_{2}^{\prime}, \ldots, x_{K}^{\prime}\right)\right.$ $\left.\mid f\left(\hat{x}_{1}^{R}, \hat{x}_{2}^{R}, \ldots, \hat{x}_{K}^{R}\right)=f\left(x_{1}, x_{2}, \ldots, x_{K}\right)\right\}$ has a diversity order two [16].

Let $m_{4}$ be a metric as defined in (18), given in the next page. The probability $P\left\{m_{1}\left(x_{1}, x_{2}, \ldots, x_{K}\right)>m_{2}\left(x_{1}^{\prime}, x_{2}^{\prime}, \ldots, x_{K}^{\prime}\right)+\log (S N R)\right.$ $\left.f\left(\hat{x}_{1}^{R}, \hat{x}_{2}^{R}, \ldots, \hat{x}_{K}^{R}\right)=f\left(x_{1}, x_{2}, \ldots, x_{K}\right)\right\}$ can be written in terms of the metrics $m_{1}$ and $m_{4}$ as in (19), which can be upper bounded as in (20) (eqns. (19) - 22) are given in the next page).

Let $\Delta x_{R}=f\left(x_{1}, x_{2}, \ldots, x_{K}\right)-x_{R}^{\prime}, \Delta x_{i}=x_{i}-x_{i}^{\prime}$. The probability $P_{H}\left\{m_{1}\left(x_{1}, x_{2}, \ldots, x_{K}\right)>m_{4}\left(x_{1}^{\prime}, x_{2}^{\prime}, \ldots, x_{K}^{\prime}, x_{R}^{\prime}\right)\right.$ $\left.\mid f\left(\hat{x}_{1}^{R}, \hat{x}_{2}^{R}, \ldots, \hat{x}_{K}^{R}\right)=f\left(x_{1}, x_{2}, \ldots, x_{K}\right)\right\}$ can be written in terms of the additive noise $z_{D_{1}}$ and $z_{D_{2}}$, as given in 21.

Let $x_{1}=\sum_{i=1}^{K} h_{S_{i} D} a_{i} \sqrt{E_{s}} \Delta x_{i}, x_{2}=\left(\sum_{i=1}^{K}\left(h_{S_{i} D} b_{i}+\right.\right.$ $\left.h_{R D} \Delta x_{R}\right) \sqrt{E_{s}}$. Also, let $\mathbf{z}_{\mathbf{D}}=\left[\begin{array}{ll}z_{D_{1}} & z_{D_{2}}\end{array}\right]$ and $\mathrm{x}=$ $\left[\begin{array}{ll}x_{1} & x_{2}\end{array}\right]^{T}$. Then (21) can be simplified as in 22, where $w=\sqrt{2} R e\left\{\mathbf{z}_{\mathbf{D}}^{*} \frac{\mathrm{x}}{\|\mathrm{x}\|}\right\}$, is distributed according to $\mathcal{N}(0,1)$. In terms of the $Q$ function, the probability $P_{H}\left\{w \leq \frac{-\log (S N R)}{\sqrt{2}\|\mathrm{x}\|}-\frac{\|\mathrm{x}\|}{\sqrt{2}}\right\}$ in (22) can be written as
$Q\left[\frac{\log (S N R)}{\sqrt{2}\|\mathrm{x}\|}+\frac{\|\mathrm{x}\|}{\sqrt{2}}\right]$. Note that $\|\mathrm{x}\|$ depends on the fade coefficients. To complete the proof, it suffices to show that $\mathbb{E}\left(Q\left[\frac{\log (S N R)}{\sqrt{2}\|\mathrm{x}\|}+\frac{\|\mathrm{x}\|}{\sqrt{2}}\right]\right)$ has a diversity order two.

The vector $\mathrm{x}$ can be written as,

$$
\begin{gathered}
\mathrm{x}=\sqrt{E_{s}} \underbrace{\left[h_{S_{1} D} h_{S_{2} D} \ldots h_{S_{K} D} h_{R D}\right]}_{\mathbf{h}} \\
\underbrace{\left[\begin{array}{lllll}
a_{1} \Delta x_{1} & a_{2} \Delta x_{2} & \ldots & a_{K} \Delta x_{K} & 0 \\
b_{1} \Delta x_{1} & b_{2} \Delta x_{2} & \ldots & b_{K} \Delta x_{K} & \Delta x_{R}
\end{array}\right]^{T}}_{\Delta \mathbf{x}} .
\end{gathered}
$$

Since $\Delta \mathbf{X} \Delta \mathbf{X}^{*}$ is Hermitian, it is unitarily diagonalizable, i.e, $\boldsymbol{\Delta} \mathbf{X} \boldsymbol{\Delta} \mathbf{X}^{*}=\mathbf{U} \boldsymbol{\Sigma} \mathbf{U}^{*}$, where $\mathbf{U}$ is unitary and $\boldsymbol{\Sigma}$ is a diagonal matrix. Since $\Delta \mathbf{X}$ has a maximum rank two, the number of non-zero diagonal entries of $\Sigma$ has to be less than or equal to two. Let $\lambda_{1}$ and $\lambda_{2}$ denote the two diagonal entries of $\Sigma$ which are possibly non-zero, with $\lambda_{1} \geq \lambda_{2}$. We have $\|\mathrm{x}\|^{2}=S N R \mathbf{h U} \boldsymbol{\Sigma} \mathbf{U}^{*} \mathbf{h}^{*}$. Let $\tilde{\mathbf{h}}=\mathbf{h} \mathbf{U}=\left[\begin{array}{lll}\tilde{h}_{1} & \tilde{h}_{2} & \tilde{h}_{3}\end{array}\right]$. The vector $\tilde{\mathbf{h}}$ has the same distribution as that of $\mathbf{h}$, since $\mathbf{U}$ is unitary.

Since the rank of $\Delta \mathbf{X}$ is at least one, $\lambda_{1}>0$. We consider the two cases where $\lambda_{2}>0$ and $\lambda_{2}=0$.

Case 1: $\lambda_{2}>0$

For this case, upper bounding $Q\left[\frac{\log (S N R)}{\sqrt{2}\|x\|}+\frac{\|x\|}{\sqrt{2}}\right]$ by 


$$
m_{4}\left(x_{1}, x_{2}, \ldots, x_{K}, x_{R}\right)=\left|y_{D_{1}}-\sum_{i=1}^{K} h_{S_{i} D} \sqrt{E_{s}} a_{i} x_{i}\right|^{2}+\left|y_{D_{2}}-\sum_{i=1}^{K} h_{S_{i} D} \sqrt{E_{s}} b_{i} x_{i}-h_{R D} \sqrt{E_{s}} x_{R}\right|^{2}+\log (S N R) .
$$

$$
\begin{aligned}
P\left\{m_{1}\left(x_{1}, x_{2}, \ldots, x_{K}\right)>m_{2}\left(x_{1}^{\prime}, x_{2}^{\prime}, \ldots, x_{K}^{\prime}\right)+\log (S N R) \mid f\left(\hat{x}_{1}^{R}, \hat{x}_{2}^{R}, \ldots, \hat{x}_{K}^{R}\right)=f\left(x_{1}, x_{2}, \ldots, x_{K}\right)\right\} \\
\quad=P\left\{m_{1}\left(x_{1}, x_{2}, \ldots, x_{K}\right)_{x_{R}^{\prime} \neq f\left(x_{1}^{\prime}, x_{2}^{\prime}, \ldots, x_{K}^{\prime}\right)} m_{4}\left(x_{1}^{\prime}, x_{2}^{\prime}, \ldots, x_{K}^{\prime}, x_{R}^{\prime}\right) \mid f\left(\hat{x}_{1}^{R}, \hat{x}_{2}^{R}, \ldots, \hat{x}_{K}^{R}\right)=f\left(x_{1}, x_{2}, \ldots, x_{K}\right)\right\} \\
\leq \sum_{x_{R}^{\prime} \neq f\left(x_{1}^{\prime}, x_{2}^{\prime}, \ldots, x_{K}^{\prime}\right)} P\left\{m_{1}\left(x_{1}, x_{2}, \ldots, x_{K}\right)>m_{4}\left(x_{1}^{\prime}, x_{2}^{\prime}, \ldots, x_{K}^{\prime}, x_{R}^{\prime}\right) \mid f\left(\hat{x}_{1}^{R}, \hat{x}_{2}^{R}, \ldots, \hat{x}_{K}^{R}\right)=f\left(x_{1}, x_{2}, \ldots, x_{K}\right)\right\} .
\end{aligned}
$$

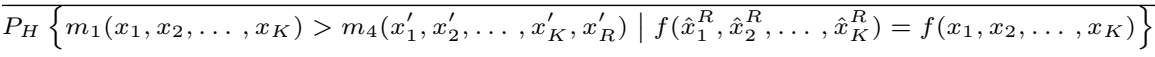

$=P_{H}\left\{\left|z_{D_{1}}\right|^{2}+\left|z_{D_{2}}\right|^{2}>\log (S N R)+\left|z_{D_{1}}+\sum_{i=1}^{K} h_{s_{i} D} \sqrt{E_{s}} a_{i} \Delta x_{i}\right|^{2}+\left|z_{D_{2}}+\sum_{i=1}^{K} h_{S_{i} D} \sqrt{E_{s}} b_{i} \Delta x_{i}+h_{R D} \sqrt{E_{s}} \Delta x_{R}\right|^{2}\right\}$.

$=P_{H}\left\{2 \operatorname{Re}\left\{\mathbf{z}_{\mathbf{D}} * \frac{\mathrm{x}}{\|\mathrm{x}\|}\right\} \leq \frac{-\log (S N R)}{\|\mathrm{x}\|}-\|\mathrm{x}\|\right\}=P_{H}\left\{w \leq \frac{-\log (S N R)}{\sqrt{2}\|\mathrm{x}\|}-\frac{\|\mathrm{x}\|}{\sqrt{2}}\right\}$.

$Q\left[\frac{\|x\|}{\sqrt{2}}\right]$, which is upper bounded by $e^{-\frac{\|x\|^{2}}{4}}$, we have

$Q\left[\frac{\log (S N R)}{\sqrt{2}\|\mathrm{x}\|}+\frac{\|\mathrm{x}\|}{\sqrt{2}}\right] \leq e^{-\frac{1}{4}\left(\lambda_{1} S N R\left|\tilde{h}_{1}\right|^{2}+\lambda_{2} S N R\left|\tilde{h}_{2}\right|^{2}\right)}$.

Taking expectation w.r.t $\left|\tilde{h}_{1}\right|$ and $\left|\tilde{h}_{2}\right|$, from $[23$, we get, $\mathbb{E}\left(Q\left[\frac{\log (S N R)}{\sqrt{2}\|x\|}+\frac{\|x\|}{\sqrt{2}}\right]\right) \leq \frac{1}{\left(1+\frac{\lambda_{1} S N R}{4}\right)\left(1+\frac{\lambda_{2} S N R}{4}\right)}$. Hence $\mathbb{E}\left(Q\left[\frac{\log (S N R)}{\sqrt{2}\|x\|}+\frac{\|x\|}{\sqrt{2}}\right]\right)$ has a diversity order two.

Case 2: $\lambda_{2}=0$

For this case $\|\mathrm{x}\|=\sqrt{\lambda_{1} S N R}\left|\tilde{h}_{1}\right|$. Hence,

$$
\begin{aligned}
& Q\left[\frac{\log (S N R)}{\sqrt{2}\|\mathrm{x}\|}+\frac{\|\mathrm{x}\|}{\sqrt{2}}\right] \\
& \quad=Q\left[\frac{\log (S N R)}{\sqrt{2} \sqrt{\lambda_{1} S N R}\left|\tilde{h}_{1}\right|}+\frac{\sqrt{\lambda_{1} S N R}\left|\tilde{h}_{1}\right|}{\sqrt{2}}\right] .
\end{aligned}
$$

Let $r=\left|\tilde{h}_{1}\right|$. Taking expectation w.r.t $r$, from 24], we get,

$$
\begin{aligned}
\mathbb{E} & \left(Q\left[\frac{\log (S N R)}{\sqrt{2}\|\mathrm{x}\|}+\frac{\|\mathrm{x}\|}{\sqrt{2}}\right]\right) \\
= & \int_{r=0}^{\infty} Q\left[\frac{\log (S N R)}{\sqrt{2} \sqrt{\lambda_{1} S N R} r}+\frac{\sqrt{\lambda_{1} S N R} r}{\sqrt{2}}\right] 2 r e^{-r^{2}} d r \\
= & \underbrace{\int_{r=0}^{\sqrt{\frac{\log (S N R)}{\lambda_{1} S N R}}} 2 Q\left[\frac{\log (S N R)}{\sqrt{2} \sqrt{\lambda_{1} S N R} r}+\frac{\sqrt{\lambda_{1} S N R} r}{\sqrt{2}}\right] r e^{-r^{2}} d r}_{I_{1}} \\
& +\underbrace{\int_{I_{2}}^{\infty}}_{r=\sqrt{\frac{\log (S N R)}{\lambda_{1} S N R}} 2 Q\left[\frac{\log (S N R)}{\sqrt{2} \sqrt{\lambda_{1} S N R} r}+\frac{\sqrt{\lambda_{1} S N R} r}{\sqrt{2}}\right] r e^{-r^{2}} d r} .
\end{aligned}
$$

In the rest of the proof, we show that the integrals $I_{1}$ and $I_{2}$ have diversity order two. Note that $\frac{\log (S N R)}{\sqrt{2} \sqrt{\lambda_{1} S N R}}+$ $\frac{\sqrt{\lambda_{1} S N R} r}{\sqrt{2}}$, as a function of $r$, attains the minimum value when $r=\sqrt{\frac{\log (S N R)}{\lambda_{1} S N R}}$ and the minimum value equals $\sqrt{2 \log (S N R)}$. Since, $Q(x)$ is a decreasing function of $x$, we have, $Q\left[\frac{\log (S N R)}{\sqrt{2} \sqrt{\lambda_{1} S N R} r}+\frac{\sqrt{\lambda_{1} S N R} r}{\sqrt{2}}\right] \leq Q[\sqrt{2 \log (S N R)}]$. Hence, we have,

$$
\begin{aligned}
I_{1} & \leq 2 Q[\sqrt{2 \log (S N R)}] \int_{r=0}^{\sqrt{\frac{\log (S N R)}{\lambda_{1} S N R}}} r e^{-r^{2}} d r \\
& \leq \frac{2}{S N R}\left(1-e^{-\frac{\log (S N R)}{\lambda_{1} S N R}}\right) .
\end{aligned}
$$

Since for small $x, e^{-x}$ can be approximated as 1 $x$, at high $S N R$, we have $I_{1} \leq \frac{2}{S N R} \frac{\log (S N R)}{\lambda_{1} S N R}$. Since $\lim _{\substack{S N R \rightarrow \infty \\ \text { least two. }}} \frac{-\log \left(\frac{2 \log (S N R)}{\lambda_{1} S N R^{2}}\right)}{\log (S N R)}=2, I_{1}$ has a diversity order at Let $r_{0}=\sqrt{\frac{\log (S N R)}{\lambda_{1} S N R}}$. The integral $I_{2}$ can be upper bounded as, $I_{2} \leq \int_{r=r_{0}}^{\infty} Q\left[\frac{\log (S N R)}{\sqrt{2} \sqrt{\lambda_{1} S N R} r}+\frac{\sqrt{\lambda_{1} S N R} r}{\sqrt{2}}\right] r d r$. Let $r^{\prime}=\frac{\log (S N R)}{\sqrt{2} \sqrt{\lambda_{1} S N R} r}+\frac{\sqrt{\lambda_{1} S N R} r}{\sqrt{2}}$. As a function of $r, r^{\prime}$ is monotonically increasing for $r \geq r_{0}$. Also, for $r \geq r_{0}, r$ can be written in terms of $r^{\prime}$ as, $r=\frac{r^{\prime}+\sqrt{r^{\prime 2}-2 \log (S N R)}}{\sqrt{2 \lambda_{1} S N R}}$. We have, $d r=d r^{\prime} \frac{1}{\sqrt{2 \lambda_{1} S N R}}\left(1+\frac{r^{\prime}}{\sqrt{r^{\prime 2}-2 \log (S N R)}}\right)$. Since $r \leq \frac{2 r^{\prime}}{\sqrt{2 \lambda_{1} S N R}}, I_{2}$ can be upper bounded in terms of $r^{\prime}$ as,

$$
\begin{aligned}
I_{2} \leq & \int_{\sqrt{2 \log (S N R)}}^{\infty} \frac{Q\left(r^{\prime}\right) r^{\prime}}{\lambda_{1} S N R}\left(1+\frac{r^{\prime}}{\sqrt{r^{\prime 2}-2 \log (S N R)}}\right) d r^{\prime} \\
= & \underbrace{\frac{1}{\lambda_{1} S N R} \int_{\sqrt{2 \log (S N R)}}^{\infty} Q\left(r^{\prime}\right) r^{\prime} d r^{\prime}}_{I_{21}} \\
& +\underbrace{\frac{1}{\lambda_{1} S N R} \int_{\sqrt{2 \log (S N R)}}^{\infty} \frac{Q\left(r^{\prime}\right) r^{\prime 2}}{\sqrt{r^{\prime 2}-2 \log (S N R)}} d r^{\prime}}_{I_{22}} .
\end{aligned}
$$

Upper bounding $Q\left(r^{\prime}\right)$ by $e^{-\frac{r^{\prime 2}}{2}}, I_{21}$ can be shown to be upper bounded as $\frac{2}{\lambda_{1} S N R^{2}}$, which falls as $S N R^{-2}$. Upper bounding $Q\left(r^{\prime}\right)$ by $e^{-\frac{r^{\prime 2}}{2}}$, and using the transformation $t=$ 
${r^{\prime}}^{2}-2 \log (S N R), I_{22}$ can be upper bounded as,

$$
\begin{aligned}
I_{22} \leq & \frac{1}{\lambda_{1} S N R^{2}} \int_{0}^{\infty} \frac{t e^{-\frac{t}{2}}}{\sqrt{t} \sqrt{t+2 \log (S N R)}} d t \\
& \quad+\frac{2 \log (S N R)}{\lambda_{1} S N R^{2}} \int_{0}^{\infty} \frac{e^{-\frac{t}{2}}}{\sqrt{t} \sqrt{t+2 \log (S N R)}} d t \\
\leq & \frac{1}{\lambda_{1} S N R^{2}} \int_{0}^{\infty} e^{-\frac{t}{2}} d t+\frac{1}{\lambda_{1} S N R^{2}} \int_{0}^{\infty} \frac{e^{-\frac{t}{2}}}{\sqrt{t}} d t \\
= & \frac{2+2 \sqrt{2 \pi} \log (S N R)}{\lambda_{1} S N R^{2}} .
\end{aligned}
$$

where the second inequality above follows from the facts that $\frac{1}{\sqrt{t+2 \log (S N R)}} \leq \frac{1}{\sqrt{t}}$ and $\frac{1}{\sqrt{t+2 \log (S N R)}} \leq 1$ for sufficiently large $S N R$. The last equality follows from the fact that $\int_{0}^{\infty} \frac{e^{-\frac{t}{2}}}{\sqrt{t}} d t=\sqrt{2} \Gamma(1 / 2)=\sqrt{2 \pi}$, where $\Gamma(z)$ is the integral, $\Gamma(z)=\int_{0}^{\infty} e^{-t} t^{z-1} d t$. Since, $\lim _{S N R \rightarrow \infty} \frac{-\log \left(\frac{2+2 \sqrt{2 \pi} \log (S N R)}{\lambda_{1} S N R^{2}}\right)}{\log (S N R)}=2, I_{22}$ has a diversity order 2. This completes the proof of Lemma 1.

Lemma 2: When the two conditions in the statement of Theorem 1 are satisfied, the probability $\quad P\left\{\left(\hat{x}_{1}^{D}, \hat{x}_{2}^{D}, \ldots, \hat{x}_{K}^{D}\right) \neq\left(x_{1}, x_{2}, \ldots, x_{K}\right) \mid\right.$ $\left.f\left(\hat{x}_{1}^{R}, \hat{x}_{2}^{R}, \ldots, \hat{x}_{K}^{R}\right)=x_{R}^{\prime}\right\}, x_{R}^{\prime} \quad \neq \quad f\left(x_{1}, x_{2}, \ldots, x_{K}\right)$, has a diversity order one.

Proof: Let $m_{4}$ denote the metric as defined in (25), given at the top of the next page. Under the condition that $R$ transmitted the wrong network coded symbol $x_{R}^{\prime}$, a decoding error occurs at $D$ only when $m_{4}\left(x_{1}, x_{2}, \ldots, x_{K}, x_{R}^{\prime}\right)>m_{4}\left(x_{1}^{\prime \prime}, x_{2}^{\prime \prime}, \ldots, x_{K}^{\prime \prime}, x_{R}^{\prime \prime}\right)$ or $m_{4}\left(x_{1}, x_{2}, \ldots, x_{K}, x_{R}^{\prime}\right)>m_{1}\left(x_{1}^{\prime \prime}, x_{2}^{\prime \prime}, \ldots, x_{K}^{\prime \prime}\right)$, for some $\left(x_{1}^{\prime \prime}, x_{2}^{\prime \prime}, \ldots, x_{K}^{\prime \prime}\right) \neq\left(x_{1}, x_{2}, \ldots, x_{K}\right)$ and $\quad x_{R}^{\prime \prime} \neq f\left(x_{1}^{\prime \prime}, x_{2}^{\prime \prime}, \ldots, x_{K}^{\prime \prime}\right)$. Hence, the probability $\quad P\left\{\left(\hat{x}_{1}^{D}, \hat{x}_{2}^{D}, \ldots, \hat{x}_{K}^{D}\right) \neq\left(x_{1}, x_{2}, \ldots, x_{K}\right)\right.$ $\left.f\left(\hat{x}_{1}^{R}, \hat{x}_{2}^{R}, \ldots, \hat{x}_{K}^{R}\right)=x_{R}^{\prime}\right\}$ can be upper bounded as in (26) (eqns. 26) - 29) are shown at the top of the next page). Using the union bound, from 26, we get 27].

Since the matrix $\left[\begin{array}{llllc}a_{1} \Delta x_{1} & a_{2} \Delta x_{2} & \ldots & a_{K} \Delta x_{K} & 0 \\ b_{1} \Delta x_{1} & b_{2} \Delta x_{2} & \ldots & b_{K} \Delta x_{K} & \Delta x_{R}\end{array}\right]^{T}$ has rank at least one for $\left(x_{1}, x_{2}, \ldots, x_{K}\right) \not$ $\left(x_{1}^{\prime \prime}, x_{2}^{\prime \prime}, \ldots, x_{K}^{\prime \prime}\right)$, where $\Delta x_{R}=x_{R}^{\prime}-$ $f\left(x_{1}^{\prime \prime}, x_{2}^{\prime \prime}, \ldots, x_{K}^{\prime \prime}\right)$, and $\Delta x_{i}=x_{i}-x_{i}^{\prime \prime}$, the probability $P\left\{m_{4}\left(x_{1}, x_{2}, \ldots, x_{K}, x_{R}^{\prime}\right)>m_{4}\left(x_{1}^{\prime \prime}, x_{2}^{\prime \prime}, \ldots, x_{K}^{\prime \prime}, x_{R}^{\prime \prime}\right)\right.$ $\left.\mid f\left(\hat{x}_{1}^{R}, \hat{x}_{2}^{R}, \ldots, \hat{x}_{K}^{R}\right)=x_{R}^{\prime}\right\}$ has a diversity order at least one.

$$
P_{H}\left\{m_{4}\left(x_{1}, x_{2}, \ldots, x_{K}, x_{R}^{\prime}\right)>m_{1}\left(x_{1}^{\prime \prime}, x_{2}^{\prime \prime}, \ldots, x_{K}^{\prime \prime}\right)\right.
$$
$\left.\mid f\left(\hat{x}_{1}^{R}, \hat{x}_{2}^{R}, \ldots, \hat{x}_{K}^{R}\right)=x_{R}^{\prime}\right\}$ can be written in terms of the additive noise $z_{D_{1}}$ and $z_{D_{2}}$, as given in 28.

Let $x_{1}=\sum_{i=1}^{K} h_{S_{i} D} a_{i} \sqrt{E_{s}} x_{i}, x_{2}=\left(\sum_{i=1}^{K} h_{S_{i} D} b_{i} x_{i}+\right.$ $\left.h_{R D} \Delta x_{R}\right) \sqrt{E_{s}}$. Also, let $\mathbf{z}_{\mathbf{D}}=\left[\begin{array}{ll}z_{D_{1}} & z_{D_{2}}\end{array}\right]$ and $\mathrm{x}=$ $\left[\begin{array}{ll}x_{1} & x_{2}\end{array}\right]^{T}$. Then (28) can be simplified as in (29), where $w=\sqrt{2} \operatorname{Re}\left\{\mathbf{z}_{\mathbf{D}}^{*} \frac{\mathrm{x}}{\|\mathrm{x}\|}\right\}$, is distributed according to $\mathcal{N}(0,1)$.
Hence,

$$
\begin{aligned}
& P_{H}\left\{w \leq \frac{\log (S N R)}{\sqrt{2}\|\mathrm{x}\|}-\frac{\|\mathrm{x}\|}{\sqrt{2}}\right\} \\
& \left.=1_{\left\{\|\mathrm{x}\|^{2}\right.} \leq \log (S N R)\right\} \\
& \quad\left(1-Q\left[\frac{\log (S N R)}{\sqrt{2}\|\mathrm{x}\|}-\frac{\|\mathrm{x}\|}{\sqrt{2}}\right]\right) \\
& \quad+1_{\left\{\|\mathrm{x}\|^{2}>\log (S N R)\right\}} Q\left[-\frac{\log (S N R)}{\sqrt{2}\|\mathrm{x}\|}+\frac{\|\mathrm{x}\|}{\sqrt{2}}\right] .
\end{aligned}
$$

Taking expectation with respect to the fade coefficients in (30), $P\left\{w \leq \frac{\log (S N R)}{\sqrt{2}\|\mathrm{x}\|}-\frac{\|\mathrm{x}\|}{\sqrt{2}}\right\}$ can be upper bounded as,

$$
\begin{gathered}
P\left\{w \leq \frac{\log (S N R)}{\sqrt{2}\|\mathrm{x}\|}-\frac{\|\mathrm{x}\|}{\sqrt{2}}\right\} \leq P\left\{\|\mathrm{x}\|^{2} \leq \log (S N R)\right\} \\
+\int_{H:\left\{\|\mathrm{x}\|^{2}>\log (S N R)\right\}} Q\left[-\frac{\log (S N R)}{\sqrt{2}\|\mathrm{x}\|}+\frac{\|\mathrm{x}\|}{\sqrt{2}}\right] d H .
\end{gathered}
$$

The vector $\mathrm{x}$ can be written as,

$$
\begin{aligned}
& \mathrm{x}=\sqrt{E_{s}} \underbrace{\left[h_{S_{1} D} h_{S_{2} D} \ldots h_{S_{K} D} h_{R D}\right]}_{\mathbf{h}} \\
& \underbrace{\left[\begin{array}{ccccc}
a_{1} \Delta x_{1} & a_{2} \Delta x_{2} & \ldots & a_{K} \Delta x_{K} & 0 \\
b_{1} \Delta x_{1} & b_{2} \Delta x_{2} & \ldots & b_{K} \Delta x_{K} & \Delta x_{R}
\end{array}\right]^{T}}_{\mathbf{\Delta} \mathbf{X}} .
\end{aligned}
$$

Since $\Delta \mathrm{X} \Delta \mathrm{X}^{*}$ is Hermitian, it is unitarily diagonalizable, i.e, $\boldsymbol{\Delta} \mathbf{X} \boldsymbol{\Delta} \mathbf{X}^{*}=\mathbf{U} \boldsymbol{\Sigma} \mathbf{U}^{*}$, where $\mathbf{U}$ is unitary and $\boldsymbol{\Sigma}$ is diagonal with $\lambda_{1}$ and $\lambda_{2}$ denoting the two possible non-zero diagonal entries, where $\lambda_{1} \geq \lambda_{2}$. Since the rank of $\Delta \mathbf{X}$ is at least one, $\lambda_{1}>0$. We have $\|x\|^{2}=S N R \mathbf{h U} \boldsymbol{\Sigma} \mathbf{U}^{*} \mathbf{h}^{*}$. Let $\tilde{\mathbf{h}}=\mathbf{h} \mathbf{U}=$ $\left[\begin{array}{lll}\tilde{h}_{1} & \tilde{h}_{2} & \tilde{h}_{3}\end{array}\right]$. The vector $\tilde{\mathbf{h}}$ has the same distribution as that of $\mathbf{h}$, since $\mathbf{U}$ is unitary. Hence, we have,

$$
\begin{aligned}
P\left\{\|\mathrm{x}\|^{2} \leq \log (S N R)\right\} & =P\left\{\lambda_{1}\left|\tilde{h}_{1}\right|^{2}+\lambda_{2}\left|\tilde{h}_{2}\right|^{2} \leq \frac{\log (S N R)}{S N R}\right\} \\
& \leq P\left\{\lambda_{1}\left|\tilde{h}_{1}\right|^{2} \leq \frac{\log (S N R)}{S N R}\right\} .
\end{aligned}
$$

Since, $\left|\tilde{h}_{1}\right|^{2}$ is exponentially distributed,

$$
P\left\{\|\mathrm{x}\|^{2} \leq \log (S N R)\right\} \leq\left(1-e^{-\frac{\log (S N R)}{\lambda_{1} S N R}}\right) .
$$

At high SNR, $1-e^{-\frac{\log (S N R)}{\lambda_{1} S N R}}$ can be approximated as $\frac{\log (S N R)}{\lambda_{1} S N R}$. $P\left\{\|\mathrm{x}\|^{2} \leq \log (S N R)\right\}$ has a diversity order at least one since $\lim _{S N R \rightarrow \infty} \frac{-\log \left(\frac{\log (S N R)}{\lambda_{1} S N R}\right)}{\log (S N R)}=1$. Since $Q(x)<$ $e^{-\frac{x^{2}}{2}}$, the integral on the right hand side of 31 can be upper bounded as,

$$
\begin{aligned}
& \int_{H:\left\{\|x\|^{2}>\log (S N R)\right\}} Q\left[-\frac{\log (S N R)}{\sqrt{2}\|\mathrm{x}\|}+\frac{\|\mathrm{x}\|}{\sqrt{2}}\right] d H \\
& \leq \int_{H:\left\{\|\mathrm{x}\|^{2}>\log (S N R)\right\}} e^{-\frac{\left(-\frac{\log (S N R)}{\|x\|^{2}}+\|x\|\right)^{2}}{4}} d H .
\end{aligned}
$$

We consider the following two cases when $\lambda_{2}>0$ and $\lambda_{2}=$ 0 .

Case 1: $\lambda_{2}>0$ 


$$
m_{4}\left(x_{1}, x_{2}, \ldots, x_{K}, x_{R}\right)=\left|y_{D_{1}}-\sum_{i=1}^{K} h_{S_{i} D} \sqrt{E_{s}} a_{i} x_{i}\right|^{2}+\left|y_{D_{2}}-\sum_{i=1}^{K} h_{S_{i} D} \sqrt{E_{s}} b_{i} x_{i}-h_{R D} \sqrt{E_{s}} x_{R}\right|^{2}+\log (S N R) .
$$

$$
\begin{aligned}
& P\left\{\left(\hat{x}_{1}^{D}, \hat{x}_{2}^{D}, \ldots, \hat{x}_{K}^{D}\right) \neq\left(x_{1}, x_{2}, \ldots, x_{K}\right) \mid f\left(\hat{x}_{1}^{R}, \hat{x}_{2}^{R}, \ldots, \hat{x}_{K}^{R}\right)=x_{R}^{\prime}\right\} \\
\quad & \sum_{\substack{\left(x_{A}^{\prime \prime}, x_{B}^{\prime \prime}\right) \in \mathcal{S}^{2} \\
\left(x_{A}^{\prime \prime}, x_{B}^{\prime \prime}\right) \neq\left(x_{1}, x_{2}, \ldots, x_{K}\right)}} P\left\{\left\{m_{4}\left(x_{1}, x_{2}, \ldots, x_{K}, x_{R}^{\prime}\right)>m_{4}\left(x_{A}^{\prime \prime}, x_{B}^{\prime \prime}, x_{R}^{\prime \prime}\right), x_{R}^{\prime \prime} \neq f\left(x_{A}^{\prime \prime}, x_{B}^{\prime \prime}\right)\right\} \cup\left\{m_{4}\left(x_{1}, x_{2}, \ldots, x_{K}, x_{R}^{\prime}\right)>m_{1}\left(x_{A}^{\prime \prime}, x_{B}^{\prime \prime}\right)\right\} \mid f\left(\hat{x}_{1}^{R}, \hat{x}_{2}^{R}, \ldots, \hat{x}_{K}^{R}\right)=x_{R}^{\prime}\right\}
\end{aligned}
$$

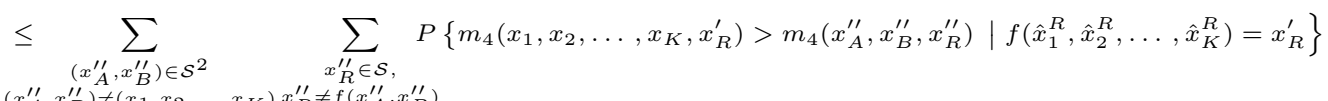
$\left(x_{A}^{\prime \prime}, x_{B}^{\prime \prime}\right) \neq\left(x_{1}, x_{2}, \ldots, x_{K}\right) x_{R}^{\prime \prime} \neq f\left(x_{A}^{\prime \prime}, x_{B}^{\prime \prime}\right)$

$$
+\sum_{\substack{\left(x_{A}^{\prime \prime}, x_{B}^{\prime \prime}\right) \in \mathcal{S}^{2} \\\left(x_{A}^{\prime \prime}, x_{B}^{\prime \prime}\right) \neq\left(x_{1}, x_{2}, \ldots, x_{K}\right)}} P\left\{m_{4}\left(x_{1}, x_{2}, \ldots, x_{K}, x_{R}^{\prime}\right)>m_{1}\left(x_{A}^{\prime \prime}, x_{B}^{\prime \prime}\right) \mid f\left(\hat{x}_{1}^{R}, \hat{x}_{2}^{R}, \ldots, \hat{x}_{K}^{R}\right)=x_{R}^{\prime}\right\}
$$

$$
\begin{aligned}
P_{H}\left\{\log (S N R)+m_{4}\left(x_{1}, x_{2}, \ldots, x_{K}, x_{R}^{\prime}\right)>m_{1}\left(x_{A}^{\prime \prime}, x_{B}^{\prime \prime}\right) \mid f\left(\hat{x}_{1}^{R}, \hat{x}_{2}^{R}, \ldots, \hat{x}_{K}^{R}\right)=x_{R}^{\prime}\right\} \\
=P_{H}\left\{\left|z_{D_{1}}\right|^{2}+\left|z_{D_{2}}\right|^{2}+\log (S N R)>\left|z_{D_{1}}+\sum_{i=1}^{K} h_{S_{i} D} \sqrt{E_{s}} a_{i} \Delta x_{i}+\right| z_{D_{2}}+\sum_{i=1}^{K} h_{S_{i} D} \sqrt{E_{s}} b_{i} \Delta x_{i}+\left.h_{R D} \Delta x_{R}\right|^{2}\right\} \\
=P_{H}\left\{2 \operatorname{Re}\left\{\mathbf{z}_{\mathbf{D}}^{*} \frac{\mathrm{x}}{\|\mathrm{x}\|}\right\} \leq \frac{\log (S N R)}{\|\mathrm{x}\|}-\|\mathrm{x}\|\right\}=P_{H}\left\{w \leq \frac{\log (S N R)}{\sqrt{2}\|\mathrm{x}\|}-\frac{\|\mathrm{x}\|}{\sqrt{2}}\right\}
\end{aligned}
$$

For this case, from the integral in (32), we get,

$$
\begin{aligned}
& \int_{H:\left\{\|\mathrm{x}\|^{2}>\log (S N R)\right\}} Q\left[-\frac{\log (S N R)}{\sqrt{2}\|\mathrm{x}\|}+\frac{\|\mathrm{x}\|}{\sqrt{2}}\right] d H \\
& \leq \int_{\left|\tilde{h}_{1}\right|^{2}=0}^{\infty} \int_{\left|\tilde{h}_{2}\right|^{2}=0}^{\infty} e^{\log (S N R)} e^{-\frac{S N R\left(\lambda_{1}\left|\tilde{h}_{1}\right|^{2}+\lambda_{2}\left|\tilde{h}_{2}\right|^{2}\right)}{4}} \\
& =\frac{S N R}{\left(1+\frac{\lambda_{1} S N R}{4}\right)\left(1+\frac{\lambda_{2} S N R}{4}\right)},
\end{aligned}
$$

$$
I \leq \int_{r>} \underbrace{\frac{\sqrt{\log (S N R)}}{\sqrt{\lambda_{1} S N R}}}_{r_{0}} e
$$

$$
e_{k_{1}}^{\left(1+\frac{\lambda_{1} S N R}{4}\right)}(r-\underbrace{\frac{\sqrt{\lambda_{1} S N R \log (S N R)}}{4+\lambda_{1} S N R}}_{k_{2}})^{2}
$$

which falls as $S N R^{-1}$ at high SNR.

Case 2: $\lambda_{2}=0$

For this case,

$$
\begin{array}{r}
I \triangleq \int_{H:\left\{\|\mathrm{x}\|^{2}>\log (S N R)\right\}} Q\left[-\frac{\log (S N R)}{\sqrt{2}\|\mathrm{x}\|}+\frac{\|\mathrm{x}\|}{\sqrt{2}}\right] f(H) d H \\
\leq \int_{\left|\tilde{h}_{1}\right|>\frac{\sqrt{\log (S N R)}}{\sqrt{\lambda_{1} S N R}}} Q\left[\frac{\sqrt{\lambda_{1} S N R\left|\tilde{h}_{1}\right|-\sqrt{\log (S N R)}}}{\sqrt{2}}\right] \\
2\left|\tilde{h}_{1}\right| e^{-\left|\tilde{h}_{1}\right|^{2}} d\left|\tilde{h}_{1}\right| .
\end{array}
$$

The above inequality follows from the fact that for $\left|\tilde{h}_{1}\right|>$ $\frac{\sqrt{\log (S N R)}}{\sqrt{\lambda_{1} S N R}},-\frac{\log (S N R)}{\sqrt{\lambda_{1} S N R}\left|\tilde{h}_{1}\right|}<-\sqrt{\log (S N R)}$. Let $r=\left|\tilde{h}_{1}\right|$. From 33 , since $Q(x)<e^{-\frac{x^{2}}{2}}$ the integral $I$ can be upper bounded as, 